\title{
Efficient and practical syntheses of three pentasaccharides core structures corresponding to $\boldsymbol{N}$-glycans
}

\author{
Yuguo Du, Meimei Zhang and Fanzuo Kong* \\ Research Center for Eco-Environmental Sciences, Chinese Academy of Sciences, P.O. Box 2871, Beijing 100085, \\ People's Republic of China \\ Dedicated to Professor Joachim Thiem on the occasion of his 60th birthday
}

Received 11 September 2000; revised 4 December 2000; accepted 14 December 2000

\begin{abstract}
Three branched pentasaccharide derivatives and one tetrasaccharide were synthesized efficiently. The advantages of this method include a one-pot facile synthesis of 3,6-differentially protected mannose building block and an efficient strategy for oligosaccharide assembly. (C) 2001 Elsevier Science Ltd. All rights reserved.
\end{abstract}

\section{Introduction}

Asparagine-linked glycoprotein oligosaccharides play a vital role in fundamental biological processes such as cell differentiation, malignant transformation, viral, bacterial and parasitic infections and protein transportations. ${ }^{1}$ They are usually divided into three major subgroups, i.e. highmannose type, complex type and hybrid type, and each of them may consist of diverse structures in living cells. ${ }^{2}$ Pentasaccharides A, B, C (Fig. 1) are typical sub-structures corresponding to the aforementioned three different types of $\mathrm{N}$-glycan, respectively. Obviously, they are the bases for the synthesis of advanced $\mathrm{N}$-glycans. Intensive research into the biological role of these carbohydrates and related glycoconjugates has led to an increased need for the practical synthesis of these core structures. Although remarkable progress has been made in the field of $\mathrm{N}$-glycan synthesis, ${ }^{3}$ further innovations are still required since the synthesis



A

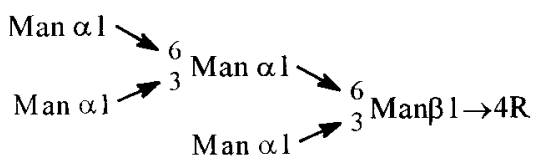

$\mathrm{C}$ remains a highly specialized and time consuming task. We present here, a very efficient and practical method ${ }^{4}$ for the synthesis of 3,6-branched oligosaccharide moieties that occur in most complex $\mathrm{N}$-glycans.

\section{Results and discussion}

To supply enough samples for glycobiology studies on the relationship between $\mathrm{N}$-glycans and diseases, we have been seeking practical methods for the synthesis of $\mathrm{N}$-glycan core structures. Scheme 1 depicts the synthesis of key synthon 2 via a one-pot manner. The transformation of allyl $\alpha$-Dmannopyranoside (1) into allyl 2,4-di- $O$-benzoyl-3-O-tertbutyldimethylsilyl-6- $O$-triphenylmethyl- $\alpha$-D-mannopyranoside (2) was successfully achieved through three sequential reactions. Thus, $\mathbf{1}$ was selectively tritylated with triphenylmethyl chloride ( $\mathrm{TrCl}, 1.25$ equiv.) in pyridine at $80^{\circ} \mathrm{C}$ for

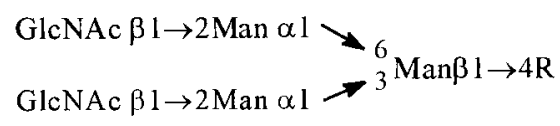

B

$$
\mathrm{R}=\mathrm{GlcNAc} \beta 1 \rightarrow 4 \mathrm{GlcNAc} \rightarrow \mathrm{Asn}
$$

Figure 1.

Keywords: carbohydrates; glycosylations; glycopeptides.

* Corresponding author. Tel.: +86-10-629-366-13; fax: +86-10-629-235-63; e-mail: fzkong@mail.rcees.ac.cn 


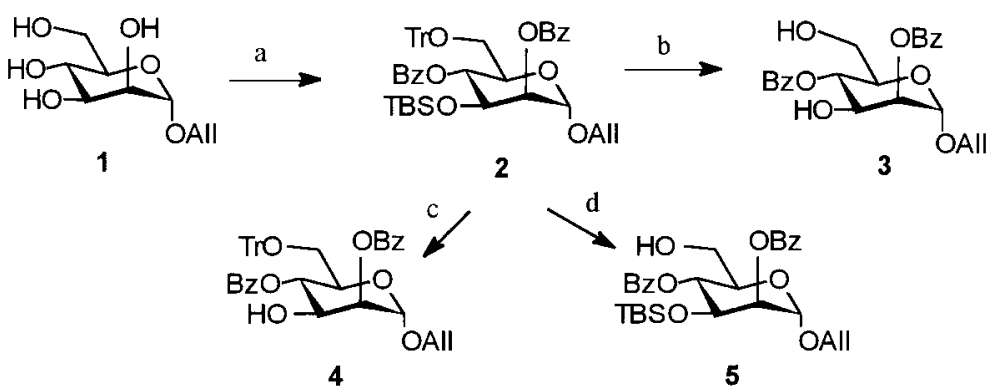

Scheme 1. Regioselective synthesis of versatile building block 2. Reaction conditions: (a) TrCl, Py, DMAP; TBSCl in DMF; BzCl in Py, 79\% in one-pot; (b) $90 \%$ TFA, 91\%; (c) TBAF, THF, 66\%; (d) $\mathrm{FeCl}_{3} \cdot 6 \mathrm{H}_{2} \mathrm{O}, \mathrm{CH}_{2} \mathrm{Cl}_{2}, 88 \%$.

$16 \mathrm{~h}$, then cooled down to $0^{\circ} \mathrm{C}$. This mixture was treated with 2 equiv. of imidazole, and finally 1.1 equiv. of tertbutylchlorodimethylsilane (TBDMSCl) in DMF was added into the reaction flask portion by portion during $2 \mathrm{~h}$. The mixture was stirred at $\mathrm{rt}$ overnight, then premixed benzoyl chloride ( 2.5 equiv.) and pyridine was added. The reaction mixture was stirred at $50^{\circ} \mathrm{C}$ overnight to afford compound $\mathbf{2}$ in $79 \%$ overall yield.

It is noteworthy that $\mathbf{2}$ could be easily transformed into a suitable acceptor for the synthesis of either symmetrical (C-3 and C-6 connected with identical sugar residues) or asymmetrical (C-3 and C-6 linked by non-identical sugar residues) trisaccharide core structure. Treatment of compound 2 with $90 \%$ trifluoroacetic acid (TFA) ${ }^{5}$ gave diol 3 in $91 \%$ yield which could be used for the synthesis of symmetrical analogs (see Scheme 2). When 2 was treated with tetrabutylammonium fluoride in tetrahedrofuran (THF), ${ }^{6} 3-\mathrm{OH}$ derivative 4 was obtained in $66 \%$ yield, and 4 could be used to synthesize $1 \rightarrow 3$ linked structure (see Scheme 3). Treatment of 2 with ferric chloride hexahydrate $\left(\mathrm{FeCl}_{3} \cdot 6 \mathrm{H}_{2} \mathrm{O}\right)^{7}$ afforded $\mathbf{5}(88 \%)$ as a useful synthon for the synthesis of $1 \rightarrow 6$ linked structure (see Scheme 4).
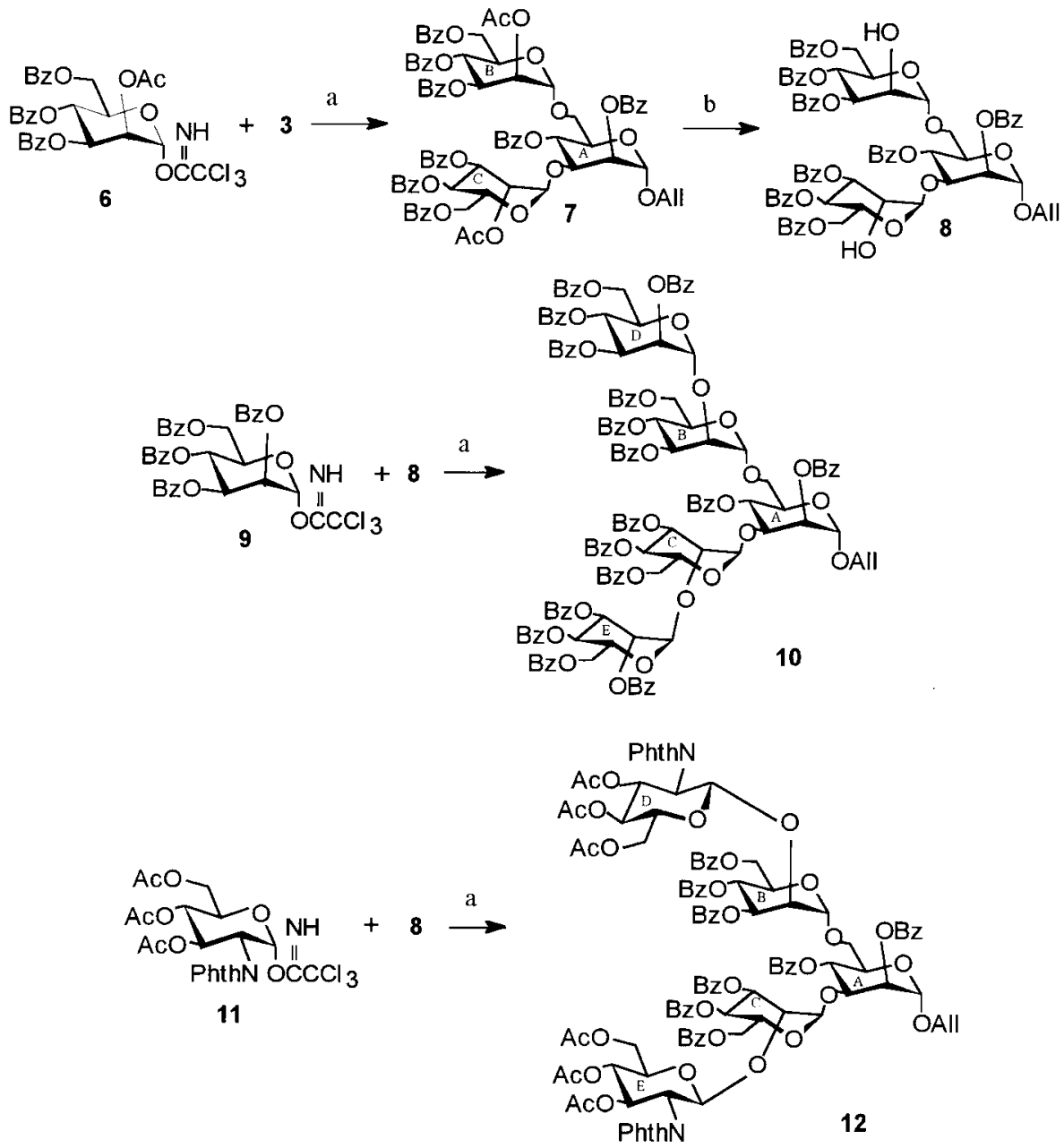

Scheme 2. Synthesis of pentasaccharides $\mathbf{1 0}$ and 12. Reaction conditions: (a) TMSOTf, $\mathrm{CH}_{2} \mathrm{Cl}_{2}, 0^{\circ} \mathrm{C}, 80 \%$ (for 7); $82 \%$ (for 10); $50 \%$ (for $\mathbf{1 2}$ ); (b) $5 \% \mathrm{HCl}$ (gas) in $\mathrm{MeOH}-\mathrm{CH}_{2} \mathrm{Cl}_{2}(1: 1), 78 \%$. 


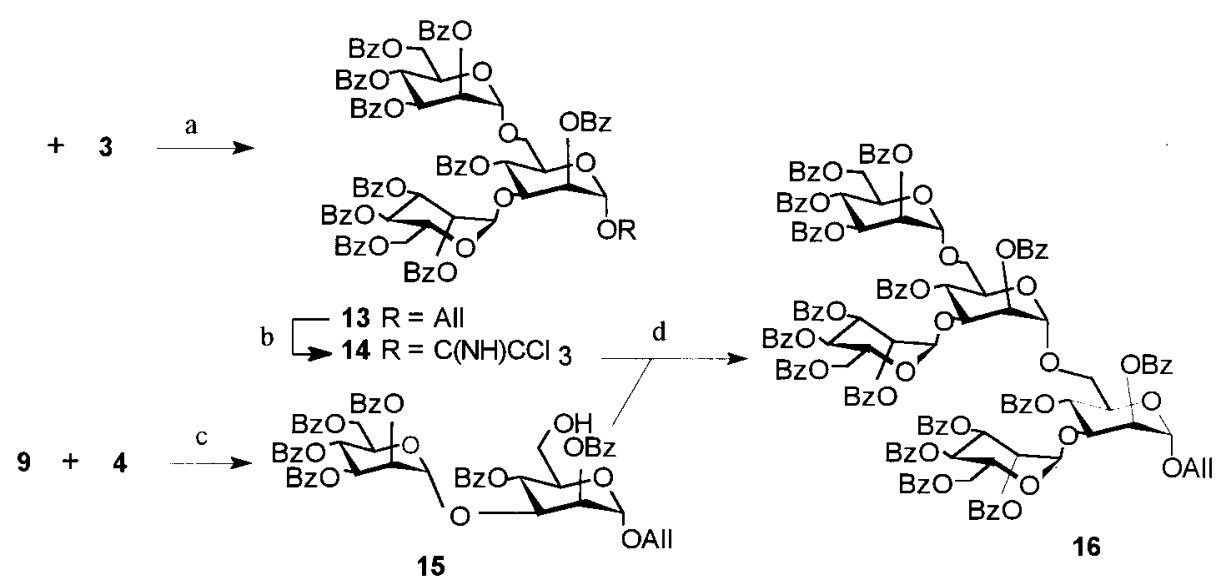

Scheme 3. Synthesis of pentasaccharide 16. Reaction conditions: (a) TMSOTf, $\mathrm{CH}_{2} \mathrm{Cl}_{2}, 0^{\circ} \mathrm{C}, 85 \%$; (b) $\mathrm{PdCl}_{2}, \mathrm{NaOAc}, 90 \% \mathrm{HOAc}$, then CCl $\mathrm{CN}, \mathrm{DBU}, 83 \%$ (2 steps); (c) TMSOTf, $\mathrm{CH}_{2} \mathrm{Cl}_{2},-15^{\circ} \mathrm{C}$, then TMSOTf (50 $\mu \mathrm{L}$ ), rt, $80 \%$; (d) TMSOTf, $\mathrm{CH}_{2} \mathrm{Cl}_{2}, 0^{\circ} \mathrm{C}, 85 \%$.

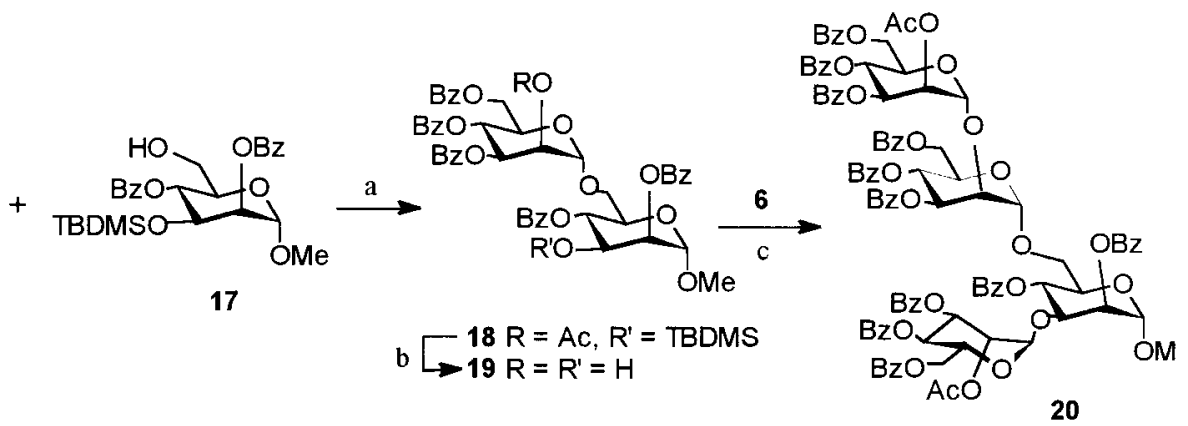

Scheme 4. Synthesis of asymmetrical tetrasaccharide 20. Reaction conditions: (a) TMSOTf, $\mathrm{CH}_{2} \mathrm{Cl}_{2}, 0^{\circ} \mathrm{C}, 84 \%$; (b) $5 \% \mathrm{HCl}(\mathrm{gas})$ in $\mathrm{MeOH}-\mathrm{CH}_{2} \mathrm{Cl}_{2}(1: 1)$, $70 \%$; (c) 2.2 equiv. of 6 , TMSOTf, $\mathrm{CH}_{2} \mathrm{Cl}_{2}, 0^{\circ} \mathrm{C}, 73 \%$.

Pentasaccharide $\mathbf{1 0}$ or $\mathbf{1 2}$ consists of three differently linked sugar residues, which necessitates a corresponding protective group pattern for these building blocks. Previous research focused on the use of benzyl ether as hydroxyl protection group. However, readily removable acyl groups are generally more attractive. We chose acetyl as a temporary protective group and benzoyl as a permanent one. Coupling of diol 3 with 2.1 equiv. of $2-O$-acetyl3,4,6-tri- $O$-benzoyl- $\alpha$-D-mannopyranosyl 2,2,2 trichloroacetimidate (6) in anhydrous $\mathrm{CH}_{2} \mathrm{Cl}_{2}$ using TMSOTf as catalyst gave symmetrical trisaccharide 7 in $80 \%$ yield. Selective deacetylation ${ }^{8}$ of 7 in the presence of benzoyl groups was carried out smoothly using $5 \% \mathrm{HCl}$ (gas) in methanol- $\mathrm{CH}_{2} \mathrm{Cl}_{2}$ (1:1) co-solvent to give deacetylated trisaccharide $\mathbf{8}$ in $78 \%$ yield. Glycosylation of trisaccharide diol 8 with donor 9 under the same reaction conditions as described in the preparation of $\mathbf{7}$ furnished pentasaccharide $\mathbf{1 0}$ in $82 \%$ yield. Similarly, coupling of $\mathbf{8}$ with 2.5 equiv. of 3,4,6-tri- $O$-acetyl-2-deoxy-2-phthalimido- $\alpha$-D-glucopyranosyl 2,2,2 trichloroacetimidate (11) afforded pentasaccharide $\mathbf{1 2}$ in $50 \%$ yield, together with some unidentified tetrasaccharides. The quick consumption of the donor $\mathbf{1 1}$ may be responsible for the low yield in this reaction.

Pentasaccharide 16, which contains both $1 \rightarrow 3$ and $1 \rightarrow 6$ linkages, best exhibits the advantages of our synthetic strategy. Coupling of diol $\mathbf{3}$ with trichloroacetimidate $\mathbf{9}$ (2.1 equiv.) in anhydrous $\mathrm{CH}_{2} \mathrm{Cl}_{2}$ using TMSOTf (10\% equiv.) as catalyst gave trisaccharide $\mathbf{1 3}$ in $85 \%$ yield. Deallylation on 13 with $\mathrm{PdCl}_{2}$ (2 equiv.) and $\mathrm{NaOAc}$ (4 equiv.) in $90 \%$ aqueous acetic acid, ${ }^{9}$ followed by anomeric Schmidt activation ${ }^{10}$ with trichloroactonitrile furnished trisaccharide donor $\mathbf{1 4}$ in $83 \%$ yield (2 steps). Convergently, coupling of donor $\mathbf{9}$ with acceptor $\mathbf{4}$ was accomplished in $40 \mathrm{~min}$ using TMSOTf (0.1 equiv.) as catalyst at $-15^{\circ} \mathrm{C}$. Trifluoacetic acid (TFA, $1 \mathrm{~mL}$ ) or more TMSOTf was added into this reaction mixture and the mixture was stirred at room temperature for $2 \mathrm{~h}$ to afford disaccharide acceptor $\mathbf{1 5}$ in one-pot (80\%). Trisaccharide donor 14 and disaccharide acceptor 15 were treated under the same glycosylation conditions affording pentasaccharide $\mathbf{1 6}$ in $85 \%$ yield. Coupled ${ }^{13} \mathrm{C}$ NMR spectrum of 10, 13 and 16 showed all ${ }^{1} J_{\mathrm{C}-1, \mathrm{H}-1}$ values in a range $170-173 \mathrm{~Hz}$, indicating the $\alpha$-linkage of mannose residues in them.

Mono-hydroxyl acceptor 17 was synthesized starting from its corresponding methyl $\alpha$-D-mannopyranoside using the same procedure as described in the preparation of $\mathbf{5}$. TMSOTf promoted coupling reaction of $\mathbf{1 7}$ with donor $\mathbf{6}$ in anhydrous $\mathrm{CH}_{2} \mathrm{Cl}_{2}$ at $0^{\circ} \mathrm{C}$ afforded disaccharide 18 in $84 \%$ yield. Simultaneous desilylation and deacetylation of 18 in the presence of benzoyl groups was carried out using $5 \% \mathrm{HCl}$ (gas) in methanol- $\mathrm{CH}_{2} \mathrm{Cl}_{2}$ (1:1) co-solvent giving disaccharide diol 19 in $70 \%$ yield. Further glycosylation of diol 19 with donor 6 under the same reaction conditions furnished asymmetrical tetrasaccharide $\mathbf{2 0}$ in $\mathbf{7 3 \%}$ yield. 


\section{Experimental}

\subsection{General methods}

Optical rotations were determined at $25^{\circ} \mathrm{C}$ with a PerkinElmer Model 241-Mc automatic polarimeter. Melting points were determined with a 'Mel-Temp' apparatus. ${ }^{1} \mathrm{H}$ NMR, ${ }^{13} \mathrm{C}$ NMR and ${ }^{1} \mathrm{H}-{ }^{1} \mathrm{H}$ COSY and ${ }^{1} \mathrm{H}-{ }^{13} \mathrm{C}$ COSY spectra were recorded with Bruker ARX 400 spectrometers for solutions in $\mathrm{CDCl}_{3}$. Chemical shifts are given in ppm downfield from internal $\mathrm{Me}_{4} \mathrm{Si}$. Mass spectra were measured using MALTI-TOF-MS with CCA as matrix or recorded with a VG PLATFORM mass spectrometer using the ESI technique to introduce the sample. IR spectra were recorded with a Hitachi 270-30 spectrometer. Thin-layer chromatography (TLC) was performed on silica gel $\mathrm{HF}_{254}$ with detection by charring with $30 \%(\mathrm{v} / \mathrm{v}) \mathrm{H}_{2} \mathrm{SO}_{4}$ in $\mathrm{MeOH}$ or in some cases by a UV detector. Column chromatography was conducted by elution of a column $\left(16 \times 240 \mathrm{~mm}^{2}\right.$, $\left.18 \times 300 \mathrm{~mm}^{2}, 35 \times 400 \mathrm{~mm}^{2}\right)$ of silica gel (100-200 mesh) with EtOAc-petroleum ether $\left(60-90^{\circ} \mathrm{C}\right)$ as the eluent. Solutions were concentrated at $<60^{\circ} \mathrm{C}$ under reduced pressure.

3.1.1. Allyl 2,4-di-O-benzoyl-3-O-tert-butyldimethylsilyl6- $O$-triphenylmethyl- $\alpha$-D-mannopyranoside (2). To a solution of allyl $\alpha$-D-mannopyranoside (1) (2.2 g, $10 \mathrm{mmol})$ in pyridine $(15 \mathrm{~mL})$ was added $\mathrm{TrCl}(3.5 \mathrm{~g}$, $12.5 \mathrm{mmol}$ ) and catalytic amount of 4-dimethylaminopyridine (DMAP). The mixture was stirred at $80^{\circ} \mathrm{C}$ for $16 \mathrm{~h}$, then cooled down to $0^{\circ} \mathrm{C}$. To the above reaction mixture was added imidazole $(1.36 \mathrm{~g}, 20 \mathrm{mmol})$ in one portion. A solution of TBDMSCl $(1.66 \mathrm{~g}, 11 \mathrm{mmol})$ in DMF $(5 \mathrm{~mL})$ was finally added portion by portion during $2 \mathrm{~h}$. The mixture was stirred at rt overnight, then a premixed solution of benzoyl chloride $(2.9 \mathrm{~mL}, 25 \mathrm{mmol})$ and pyridine $(3 \mathrm{~mL})$ was added. The reaction mixture was stirred at $50^{\circ} \mathrm{C}$ overnight, and then poured into ice-cold water, extracted with EtOAc. The organic phase was concentrated to dryness by repeating co-evaporation with toluene. The residue was subjected to column chromatography on silica gel with petroleum etherEtOAc as the eluent (12:1) to give 2 as a syrup (6.19 g, $79 \%) ;[\alpha]_{\mathrm{D}}{ }^{25}=-31\left(c 1, \mathrm{CHCl}_{3}\right) ; \nu_{\max }$ (liquid film): 2932, 1734,$1602 ; \delta_{\mathrm{H}}\left(400 \mathrm{MHz}, \mathrm{CDCl}_{3}\right)-0.15,0.04(2 \mathrm{~s}, 2 \times 3 \mathrm{H}$, $\left.\left(\mathrm{CH}_{3}\right)^{2} \mathrm{Si}\right), 0.62$, (s, 9H, $\left.t-\mathrm{Bu}\right), 3.75\left(\mathrm{dd}, 1 \mathrm{H}, J_{5,6 \mathrm{a}}=1.8 \mathrm{~Hz}\right.$, $\left.J_{6 \mathrm{a}, 6 \mathrm{~b}}=11.2 \mathrm{~Hz}, \mathrm{H}-6 \mathrm{a}\right), 3.84\left(\mathrm{dd}, 1 \mathrm{H}, J_{5,6 \mathrm{~b}}=5.3 \mathrm{~Hz}, J_{6 \mathrm{a}, 6 \mathrm{~b}}=\right.$ $11.2 \mathrm{~Hz}, \mathrm{H}-6 \mathrm{~b}$ ), 3.98 (ddd, $J_{5,6 \mathrm{a}}=1.8 \mathrm{~Hz}, J_{5,6 \mathrm{~b}}=5.3 \mathrm{~Hz}$, $\left.J_{4,5}=9.9 \mathrm{~Hz}, 1 \mathrm{H}, \mathrm{H}-5\right), 4.09\left(\mathrm{~m}, 1 \mathrm{H}, \mathrm{CH}_{2}=\mathrm{CH}-\mathrm{CH}_{2}-\right)$, $4.28\left(\mathrm{~m}, 1 \mathrm{H}, \mathrm{CH}_{2}=\mathrm{CH}-\mathrm{CH}_{2}-\right), 4.38\left(\mathrm{dd}, 1 \mathrm{H}, J_{2,3}=\right.$ $\left.3.5 \mathrm{~Hz}, J_{3,4}=9.4 \mathrm{~Hz}, \mathrm{H}-3\right), 5.03$ (d, 1H, $\left.J_{1,2}=1.4 \mathrm{~Hz}, \mathrm{H}-1\right)$, 5.24-5.36 (m, 2H, $\left.\mathrm{CH}_{2}=\mathrm{CH}-\mathrm{CH}_{2}-\right), 5.40$ (dd, $J_{1,2}=$ $\left.1.4 \mathrm{~Hz}, J_{2,3}=3.5 \mathrm{~Hz}, 1 \mathrm{H}, \mathrm{H}-2\right), 5.73\left(\mathrm{dd}, 1 \mathrm{H}, J_{3,4}=9.4 \mathrm{~Hz}\right.$, $\left.J_{4,5}=9.9 \mathrm{~Hz}, \mathrm{H}-4\right), 5.91-5.96\left(\mathrm{~m}, 1 \mathrm{H}, \mathrm{CH}_{2}=\mathrm{CH}-\mathrm{CH}_{2}-\right)$, 7.20-8.30 (m, 25H, Ph). Anal. Calcd for $\mathrm{C}_{48} \mathrm{H}_{52} \mathrm{O}_{8} \mathrm{Si}: \mathrm{C}$, 73.47; H, 6.63. Found: C, 73.51; H, 6.58.

3.1.2. Allyl 2,4-di-O-benzoyl- $\alpha$-D-mannopyranoside (3). Compound 2 (2.5 g, $3.19 \mathrm{mmol})$ was dissolved into $90 \%$ aqueous TFA $(15 \mathrm{~mL})$ and the solution was stirred at $\mathrm{rt}$ for $4 \mathrm{~h}$. Toluene $(50 \mathrm{~mL})$ was added and then the solvents were evaporated in vacuo to give a residue, which was purified by silica gel column chromatography (petroleum ether-EtOAc, $1: 1)$ to give 3 as a white foam $(1.24 \mathrm{~g}$,
91\%); $[\alpha]_{\mathrm{D}}{ }^{25}=-26$ (c 1, $\left.\mathrm{CHCl}_{3}\right) ; \nu_{\max }$ (liquid film): 3443, 2925,$1608 ; \delta_{\mathrm{H}}\left(400 \mathrm{MHz}, \mathrm{CDCl}_{3}\right) 3.74\left(\mathrm{dd}, 1 \mathrm{H}, J_{5,6 \mathrm{a}}=\right.$ $\left.4.1 \mathrm{~Hz}, J_{6 \mathrm{a}, 6 \mathrm{~b}}=12.6 \mathrm{~Hz}, \mathrm{H}-6 \mathrm{a}\right), 3.81\left(\mathrm{dd}, 1 \mathrm{H}, J_{5,6 \mathrm{~b}}=2.3 \mathrm{~Hz}\right.$, $\left.J_{6 \mathrm{a}, 6 \mathrm{~b}}=12.6 \mathrm{~Hz}, \mathrm{H}-6 \mathrm{~b}\right), 3.98$ (ddd, $1 \mathrm{H}, \quad J_{5,6 \mathrm{~b}}=2.3 \mathrm{~Hz}$, $\left.J_{5,6 \mathrm{a}}=4.1 \mathrm{~Hz}, J_{4,5}=9.9 \mathrm{~Hz}, \mathrm{H}-5\right), 4.09\left(\mathrm{~m}, 1 \mathrm{H}, \mathrm{CH}_{2}=\mathrm{CH}-\right.$ $\left.\mathrm{CH}_{2}-\right), 4.26\left(\mathrm{~m}, 1 \mathrm{H}, \mathrm{CH}_{2}=\mathrm{CH}-\mathrm{CH}_{2}-\right), 4.46(\mathrm{dd}, 1 \mathrm{H}$, $\left.J_{2,3}=3.5 \mathrm{~Hz}, J_{3,4}=9.9 \mathrm{~Hz}, \mathrm{H}-3\right), 5.10\left(\mathrm{~d}, 1 \mathrm{H}, J_{1,2}=1.6 \mathrm{~Hz}\right.$, $\mathrm{H}-1), 5.25-5.34\left(\mathrm{~m}, 2 \mathrm{H}, \mathrm{CH}_{2}=\mathrm{CH}-\mathrm{CH}_{2}-\right), 5.43(\mathrm{dd}, 1 \mathrm{H}$, $\left.J_{2,3}=3.5 \mathrm{~Hz}, J_{1,2}=1.6 \mathrm{~Hz}, \mathrm{H}-2\right), 5.51\left(\mathrm{t}, 1 \mathrm{H}, J_{3,4}=9.9 \mathrm{~Hz}\right.$, $\left.J_{4,5}=9.9 \mathrm{~Hz}, \mathrm{H}-4\right), 5.91-5.96\left(\mathrm{~m}, 1 \mathrm{H}, \mathrm{CH}_{2}=\mathrm{CH}-\mathrm{CH}_{2}-\right)$, 7.25-8.10 (m, $10 \mathrm{H}, \mathrm{Ph})$. Anal. Calcd for $\mathrm{C}_{23} \mathrm{H}_{24} \mathrm{O}_{8}: \mathrm{C}$, 64.48; H, 5.61. Found: C, 64.40; H, 5.66.

3.1.3. Allyl 2-O-acetyl-3,4,6-tri- $O$-benzoyl- $\alpha$-D-mannopyranosyl-(1 $\rightarrow 3)$-[2-O-acetyl-3,4,6-tri- $O$-benzoyl- $\alpha-\mathrm{D}-$ mannopyranosyl-( $\rightarrow$ 6)]-2,4-di- $O$-benzoyl- $\alpha$-D-mannopyranoside (7). To a cooled solution $\left(0^{\circ} \mathrm{C}\right)$ of $\mathbf{3}(2.1 \mathrm{~g}$, $4.91 \mathrm{mmol})$ and $6(6.99 \mathrm{~g}, 10.3 \mathrm{mmol})$ in anhydrous $\mathrm{CH}_{2} \mathrm{Cl}_{2}(80 \mathrm{~mL})$ was added TMSOTf $(40 \mu \mathrm{L}, 0.22 \mathrm{mmol})$. The mixture was stirred at this temperature for $2 \mathrm{~h}$, and then quenched with $\mathrm{Et}_{3} \mathrm{~N}$ (2 drops). The solvents were evaporated in vacuo to give a residue, which was purified by silica gel column chromatography (petroleum ether-EtOAc, $1.5: 1)$ to give trisaccharide 7 as a syrup $(5.73 \mathrm{~g}, 80 \%)$; $[\alpha]_{\mathrm{D}}{ }^{25}=-12\left(c 1, \mathrm{CHCl}_{3}\right) ; \nu_{\max }$ (liquid film): 3442, 2926, $1730,1452,1270,1110,1070,711 \mathrm{~cm}^{-1} ; \delta_{\mathrm{H}}(400 \mathrm{MHz}$, $\left.\mathrm{CDCl}_{3}\right) 1.882,2.095\left(2 \mathrm{~s}, 6 \mathrm{H}, 2 \mathrm{CH}_{3} \mathrm{CO}\right), 3.726(\mathrm{dd}, 1 \mathrm{H}$, $\left.J_{5,6 \mathrm{a}}=1.8 \mathrm{~Hz}, J_{6 \mathrm{a}, 6 \mathrm{~b}}=10.6 \mathrm{~Hz}, \mathrm{H}-6 \mathrm{a}^{\mathrm{A}}\right), 4.080-4.190(\mathrm{~m}, 2 \mathrm{H}$, $\mathrm{H}-6 \mathrm{~b}^{\mathrm{A}}$, one proton of $\mathrm{CH}_{2}=\mathrm{CH}-\mathrm{CH}_{2}-$ ), 4.285 (ddd, $1 \mathrm{H}$, $\left.J_{5,6 \mathrm{a}}=1.8 \mathrm{~Hz}, \quad J_{5,6 \mathrm{~b}}=3.5 \mathrm{~Hz}, \quad J_{4,5}=9.7 \mathrm{~Hz}, \quad 1 \mathrm{H}, \quad \mathrm{H}-5^{\mathrm{A}}\right)$, $4.300-4.550\left(\mathrm{~m}, 7 \mathrm{H}, \mathrm{H}-5^{\mathrm{B}, \mathrm{C}}, 2 \times \mathrm{H}-6^{\mathrm{B}, \mathrm{C}}\right.$, one proton of $\left.\mathrm{CH}_{2}=\mathrm{CH}-\mathrm{CH}_{2}-\right), 4.617\left(\mathrm{dd}, 1 \mathrm{H}, \quad J_{2,3}=3.3 \mathrm{~Hz}, \quad J_{3,4}=\right.$ $\left.9.7 \mathrm{~Hz}, \mathrm{H}-3^{\mathrm{A}}\right), 4.979\left(\mathrm{~d}, 1 \mathrm{H}, J_{1,2}=1.6 \mathrm{~Hz}, \mathrm{H}_{-} 1^{\mathrm{B}}\right), 5.110-$ $5.130\left(\mathrm{~m}, 2 \mathrm{H}, \mathrm{H}-1^{\mathrm{A}}, \mathrm{H}-2^{\mathrm{C}}\right), 5.189\left(\mathrm{~d}, 1 \mathrm{H}, J_{1,2}=1.8 \mathrm{~Hz}\right.$, $\mathrm{H}-1^{\mathrm{C}}$ ), 5.290-5.507 (m, 2H, $\mathrm{CH}_{2}=\mathrm{CH}-\mathrm{CH}_{2}-$ ), 5.513 (dd, $\left.1 \mathrm{H}, \quad J_{1,2}=1.6 \mathrm{~Hz}, J_{2,3}=2.9 \mathrm{~Hz}, \mathrm{H}-2^{\mathrm{B}}\right), 5.582(\mathrm{dd}, 1 \mathrm{H}$, $\left.J_{2,3}=3.3 \mathrm{~Hz}, J_{3,4}=9.8 \mathrm{~Hz}, \mathrm{H}-3^{\mathrm{C}}\right), 5.690\left(\mathrm{dd}, 1 \mathrm{H}, J_{1,2}=\right.$ $\left.1.6 \mathrm{~Hz}, J_{2,3}=3.6 \mathrm{~Hz}, \mathrm{H}-2^{\mathrm{A}}\right), 5.793\left(\mathrm{t}, 1 \mathrm{H}, J_{3,4}=9.8 \mathrm{~Hz}\right.$, $\left.J_{4,5}=9.8 \mathrm{~Hz}, \mathrm{H}-4^{\mathrm{C}}\right), 5.841\left(\mathrm{t}, 1 \mathrm{H}, J_{3,4}=9.7 \mathrm{~Hz}, J_{4,5}=9.7 \mathrm{~Hz}\right.$, $\left.\mathrm{H}-4^{\mathrm{A}}\right), 5.865-5.890\left(\mathrm{~m}, 3 \mathrm{H}, \mathrm{H}-3^{\mathrm{B}}, \mathrm{H}-4^{\mathrm{B}}, \mathrm{CH}_{2}=\mathrm{CH}-\mathrm{CH}_{2}-\right)$, 7.252-8.093 (m, 40H, Ph). Anal. Calcd for $\mathrm{C}_{81} \mathrm{H}_{72} \mathrm{O}_{26}: \mathrm{C}$, 66.58; H, 4.93. Found: C, 66.61; H, 4.94.

3.1.4. Allyl 3,4,6-tri- $O$-benzoyl- $\alpha$-D-mannopyranosyl$(1 \rightarrow 3)$-[3,4,6-tri-O-benzoyl- $\alpha$-D-mannopyranosyl$(1 \rightarrow 6)$ ]-2,4-di- $O$-benzoyl- $\alpha$-D-mannopyranoside $(8)$. To a cooled solution $\left(0^{\circ} \mathrm{C}\right)$ of $7(720 \mathrm{mg}, 0.49 \mathrm{mmol})$ in $\mathrm{CH}_{2} \mathrm{Cl}_{2}$ $(10 \mathrm{~mL})$ and $\mathrm{MeOH}(10 \mathrm{~mL})$ was added acetyl chloride (1 mL). The mixture was stirred at rt overnight, then diluted with $\mathrm{CH}_{2} \mathrm{Cl}_{2}(200 \mathrm{~mL})$, neutralized with $\mathrm{Et}_{3} \mathrm{~N}$ and washed with water. The organic phase was dried over anhydrous $\mathrm{Na}_{2} \mathrm{SO}_{4}$ and concentrated. The residue was purified on a silica gel column using EtOAc-petroleum ether $(1: 2)$ as the elutant giving 8 as a syrup (526 mg, 78\%); $[\alpha]_{\mathrm{D}}{ }^{25}=-6$ (c 1, $\left.\mathrm{CHCl}_{3}\right) ; \nu_{\max }$ (liquid film): 3444, 2960, 1731, 1603; $\delta_{\mathrm{H}}$ (400 MHz, $\mathrm{CDCl}_{3}$ ) 2.300-2.500 (br d, 2H, 2OH), 3.797 (dd, $\left.1 \mathrm{H}, J_{5,6 \mathrm{a}}=1.2 \mathrm{~Hz}, J_{6 \mathrm{a}, 6 \mathrm{~b}}=10.8 \mathrm{~Hz}, \mathrm{H}-6 \mathrm{a}^{\mathrm{A}}\right), 4.05-4.460(\mathrm{~m}$,

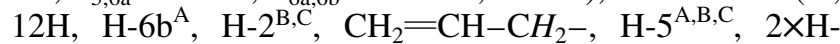
$\left.6^{\mathrm{B}, \mathrm{C}}\right), 4.618\left(\mathrm{dd}, 1 \mathrm{H}, J_{2,3}=3.4 \mathrm{~Hz}, J_{3,4}=8.7 \mathrm{~Hz}, \mathrm{H}-3^{\mathrm{A}}\right)$, $5.070\left(\mathrm{bs}, 1 \mathrm{H}, \mathrm{H}-1^{\mathrm{B}}\right), 5.154\left(\mathrm{~d}, 1 \mathrm{H}, J_{1,2}=2.0 \mathrm{~Hz}, \mathrm{H}-1^{\mathrm{A}}\right)$, 5.229 (bs, $\left.1 \mathrm{H}, \mathrm{H}-1^{\mathrm{C}}\right), 5.260-5.440\left(\mathrm{~m}, 2 \mathrm{H}, \mathrm{CH}_{2}=\mathrm{CH}-\right.$ $\left.\mathrm{CH}_{2}-\right), 5.450\left(\mathrm{dd}, 1 \mathrm{H}, J_{2,3}=3.1 \mathrm{~Hz}, J_{3,4}=9.7 \mathrm{~Hz}, \mathrm{H}-3^{\mathrm{C}}\right)$, $5.673\left(\mathrm{dd}, 1 \mathrm{H}, J_{1,2}=2.0 \mathrm{~Hz}, J_{2,3}=3.4 \mathrm{~Hz}, \mathrm{H}-2^{\mathrm{A}}\right), 5.714(\mathrm{dd}$, 
$\left.1 \mathrm{H}, \quad J_{2,3}=3.1 \mathrm{~Hz}, J_{3,4}=10.0 \mathrm{~Hz}, \quad \mathrm{H}-3^{\mathrm{B}}\right), 5.841 \quad(\mathrm{t}, \quad 1 \mathrm{H}$, $\left.J_{3,4}=9.7 \mathrm{~Hz}, \quad J_{4,5}=9.7 \mathrm{~Hz}, \quad \mathrm{H}-4^{\mathrm{C}}\right), 5.865 \quad\left(\mathrm{t}, 1 \mathrm{H}, J_{3,4}=\right.$ $\left.10.0 \mathrm{~Hz}, J_{4,5}=10.0 \mathrm{~Hz}, \mathrm{H}-4^{\mathrm{B}}\right), 5.930\left(\mathrm{t}, 1 \mathrm{H}, J_{3,4}=8.7 \mathrm{~Hz}\right.$, $\left.J_{4,5}=8.7 \mathrm{~Hz}, \mathrm{H}-4^{\mathrm{A}}\right), 5.955\left(\mathrm{~m}, 1 \mathrm{H}, \mathrm{CH}_{2}=\mathrm{CH}-\mathrm{CH}_{2}-\right)$, 7.258-8.201 (m, 40H, Ph). Anal. Calcd for $\mathrm{C}_{77} \mathrm{H}_{68} \mathrm{O}_{24}: \mathrm{C}$, 67.15; H, 4.94. Found: C, 67.09; H, 4.99.

3.1.5. Allyl 2,3,4,6-tetra- $O$-benzoyl- $\alpha$-D-mannopyranosyl-( $1 \rightarrow 2)-3,4,6$-tri- $O$-benzoyl- $\alpha$-D-mannopyranosyl$(1 \rightarrow 6)$-[2,3,4,6-tetra- $O$-benzoyl- $\alpha$-D-mannopyranosyl$(1 \rightarrow 2)-3,4,6$-tri- $O$-benzoyl- $\alpha$-D-mannopyranosyl- $(1 \rightarrow 3)]$ 2,4-di- $\boldsymbol{O}$-benzoyl- $\alpha$-D-mannopyranoside (10). To a cooled solution $\left(0^{\circ} \mathrm{C}\right)$ of $\mathbf{8}(200 \mathrm{mg}, 0.14 \mathrm{mmol})$ and $9(290 \mathrm{mg}$, $0.39 \mathrm{mmol})$ in anhydrous $\mathrm{CH}_{2} \mathrm{Cl}_{2}(7 \mathrm{~mL})$ was added TMSOTf $(5 \mu \mathrm{L}, 0.028 \mathrm{mmol})$. The mixture was stirred at this temperature for $2 \mathrm{~h}$, and then quenched with $\mathrm{Et}_{3} \mathrm{~N}$ (1 drop). The solvents were evaporated in vacuo to give a residue, which was purified by silica gel column chromatography twice (petroleum ether-EtOAc, 2:1 and toluenepetroleum ether-EtOAc, 0.75:1.5:1.0) to give pentasaccharide 10 as white solid $(290 \mathrm{mg}, 82 \%) ;[\alpha]_{\mathrm{D}}{ }^{25}=-49\left(c 1, \mathrm{CHCl}_{3}\right)$; $\nu_{\max }$ (liquid film): $3440,2958,1730,1603,1452,1270,1110$, $1071 ; \delta_{\mathrm{H}}\left(400 \mathrm{MHz}, \mathrm{CDCl}_{3}\right) 3.647\left(\mathrm{dd}, 1 \mathrm{H}, J_{5,6 \mathrm{a}}<1 \mathrm{~Hz}\right.$, $\left.J_{6 \mathrm{a}, 6 \mathrm{~b}}=10.4 \mathrm{~Hz}, \quad \mathrm{H}-6 \mathrm{a}^{\mathrm{A}}\right), \quad 3.987 \quad\left(\mathrm{dd}, \quad 1 \mathrm{H}, \quad J_{5,6 \mathrm{~b}}=5.0 \mathrm{~Hz}\right.$, $\left.J_{6 \mathrm{a}, 6 \mathrm{~b}}=10.4 \mathrm{~Hz}, \mathrm{H}-6 \mathrm{~b}^{\mathrm{A}}\right), 4.032$ (bs, $1 \mathrm{H}, \mathrm{H}-2^{\mathrm{C}}$ ), 4.179-4.230 $\left(\mathrm{m}, 3 \mathrm{H}, \mathrm{H}-5^{\mathrm{A}}, J=5.1 \mathrm{~Hz}, 12.0 \mathrm{~Hz}, 2 \mathrm{H}-6\right), 4.319$ (bs, $1 \mathrm{H}$, $\left.\mathrm{H}-2^{\mathrm{B}}\right), 4.320-4.500(\mathrm{~m}, 6 \mathrm{H}), 4.502\left(\mathrm{~s}, 1 \mathrm{H}, \mathrm{H}-1^{\mathrm{E}}\right), 4.505-$ $4.635(\mathrm{~m}, 6 \mathrm{H}), 4.640\left(\mathrm{dd}, 1 \mathrm{H}, J_{2,3}=2.5 \mathrm{~Hz}, J_{3,4}=9.9 \mathrm{~Hz}\right.$, $\left.\mathrm{H}-3^{\mathrm{A}}\right), 5.090\left(\mathrm{~s}, 1 \mathrm{H}, \mathrm{H}-1^{\mathrm{D}}\right), 5.184\left(\mathrm{~s}, 1 \mathrm{H}, \mathrm{H}-1^{\mathrm{A}}\right), 5.205(\mathrm{~s}$, $\left.1 \mathrm{H}, \mathrm{H}-1^{\mathrm{B}}\right), 5.312,5.550(2 \mathrm{dd}, 2 \mathrm{H}, J=1.2 \mathrm{~Hz}, 11.2,16.8 \mathrm{~Hz}$, $\left.\mathrm{CH}_{2}=\mathrm{CH}-\mathrm{CH}_{2}-\right), 5.481\left(\mathrm{~s}, 1 \mathrm{H}, \mathrm{H}-1^{\mathrm{C}}\right), 5.563\left(\mathrm{bs}, 1 \mathrm{H}, \mathrm{H}-2^{\mathrm{E}}\right)$, $5.688\left(\mathrm{dd}, J_{23}=2.1 \mathrm{~Hz}, J_{3,4}=10.6 \mathrm{~Hz}, \mathrm{H}-3^{\mathrm{C}}\right), 5.741(\mathrm{bs}, 1 \mathrm{H}$, $\left.\mathrm{H}-2^{\mathrm{A}}\right), 5.825\left(\mathrm{~s}, 1 \mathrm{H}, \mathrm{H}-2^{\mathrm{D}}\right), 5.827-6.005\left(\mathrm{~m}, 8 \mathrm{H}, \mathrm{H}-4^{\mathrm{A}}, \mathrm{H}-3^{\mathrm{E}}\right.$ $\left.\mathrm{H}-4^{\mathrm{E}}, \mathrm{H}-4^{\mathrm{C}}, \mathrm{H}-3^{\mathrm{B}}, \mathrm{H}-4^{\mathrm{B}}, \mathrm{H}-3^{\mathrm{D}}, \mathrm{CH}_{2}=\mathrm{CH}-\mathrm{CH}_{2}-\right), 6.095(\mathrm{t}$, $\left.1 \mathrm{H}, J_{3,4}=10.1 \mathrm{~Hz}, J_{4,5}=10.1 \mathrm{~Hz}, \mathrm{H}-4^{\mathrm{D}}\right), 7.245-8.140(\mathrm{~m}, 80 \mathrm{H}$, $\mathrm{Ph}) . \delta_{\mathrm{C}}\left(100 \mathrm{MHz}, \mathrm{CDCl}_{3}\right) 166.31,166.19$ (2C), 166.00, $165.94,165.57,165.50,165.35,165.27,165.23,165.03$, $164.94,164.88,164.83,164.80,164.51$ (16PhCO), 118.89 $\left(\mathrm{CH}_{2}=\mathrm{CH}-\mathrm{CH}_{2}-\right), 100.00,99.72,99.39,98.21,96.66(5 \mathrm{C}-$ $\left.1,{ }^{1} J_{\mathrm{C}-1, \mathrm{H}-1}=169,170,170,171,173 \mathrm{~Hz}\right), 78.4,77.47,77.21$, 75.21, 71.96, 70.57, 70.07, 69.89, 69.74 (3C), 69.63, 69.53, 69.47, 69.23, $68.70\left(\mathrm{CH}_{2}=\mathrm{CH}-\mathrm{CH}_{2}-\right), 68.75$ (2C), 67.19, $67.13,66.61,66.37,63.65,63.50,62.82,62.62$ (sugar carbons). MALDI-TOF MS: M+Na calcd: 2555, found: 2555.62; Anal. Calcd for $\mathrm{C}_{145} \mathrm{H}_{120} \mathrm{O}_{42}$ : C, 68.72; H, 4.94. Found: C, 68.76; H, 4.88 .

3.1.6. Allyl 3,4,6-tri- $O$-acetyl-2-deoxy-2-phthalimido- $\beta$ D-glucopyranosyl- $(1 \rightarrow 2)-3,4,6$-tri- $O$-benzoyl- $\alpha$-D-mannopyranosyl- $(1 \rightarrow 6)$-[3,4,6-tri- $O$-acetyl-2-deoxy-2-phthalimido- $\beta$-D-glucopyranosyl- $(1 \rightarrow 2)-3,4,6$-tri- $O$-benzoyl$\alpha$-D-mannopyranosyl- $(1 \rightarrow 3)]$-2,4-di- $O$-benzoyl- $\alpha$-Dmannopyranoside (12). To a cooled solution $\left(0^{\circ} \mathrm{C}\right)$ of 8 $(200 \mathrm{mg}, \quad 0.14 \mathrm{mmol})$ and $11(208 \mathrm{mg}, 0.36 \mathrm{mmol})$ in anhydrous $\mathrm{CH}_{2} \mathrm{Cl}_{2}(5 \mathrm{~mL})$ was added TMSOTf $(3 \mu \mathrm{L}$, $0.016 \mathrm{mmol})$. The mixture was stirred at this temperature for $2 \mathrm{~h}$, and then quenched with $\mathrm{Et}_{3} \mathrm{~N}$ (1 drop). The solvents were evaporated in vacuo to give a residue, which was purified by silica gel column chromatography twice (petroleum ether-EtOAc, 1.0:1 and toluene-petroleum ether-EtOAc, 0.2:1.0:1.0) to give pentasaccharide 12 as a syrup $(167 \mathrm{mg}, 50 \%) ;[\alpha]_{\mathrm{D}}{ }^{25}=-48\left(c 1, \mathrm{CHCl}_{3}\right) ; \nu_{\max }$ (liquid film): $3442,2932,1743,1455,1259 ; \quad \delta_{\mathrm{H}}$ $\left(400 \mathrm{MHz}, \mathrm{CDCl}_{3}\right) 1.780,1.821,1.834,1.852,1.964$, $1.974\left(6 \mathrm{~s}, 6 \times 3 \mathrm{H}, 6 \mathrm{CH}_{3} \mathrm{CO}\right), 3.370\left(\mathrm{dd}, 1 \mathrm{H}, J_{5,6 \mathrm{a}}=1.6 \mathrm{~Hz}\right.$, $\left.J_{6 \mathrm{a}, 6 \mathrm{~b}}=11.4 \mathrm{~Hz}, \quad \mathrm{H}-6 \mathrm{a}^{\mathrm{A}}\right), 3.529\left(\mathrm{dd}, \quad 1 \mathrm{H}, \quad J_{5,6 \mathrm{~b}}=3.7 \mathrm{~Hz}\right.$, $\left.J_{6 \mathrm{a}, 6 \mathrm{~b}}=11.4 \mathrm{~Hz}, \quad \mathrm{H}-6 \mathrm{~b}^{\mathrm{A}}\right), \quad 3.631 \quad\left(\mathrm{dd}, \quad 1 \mathrm{H}, \quad J_{5,6}=1.1 \mathrm{~Hz}\right.$, $J_{6 \mathrm{a}, 6 \mathrm{~b}}=10.8 \mathrm{~Hz}, \mathrm{H}-6$ ), 3.620-3.690 (m, 2H, H-6), 3.702$3.780(\mathrm{~m}, 2 \mathrm{H}, \mathrm{H}-5, \mathrm{H}-6), 3.800\left(\mathrm{dd}, 1 \mathrm{H}, J_{5,6}=3.8 \mathrm{~Hz}\right.$, $\left.J_{6 \mathrm{a}, 6 \mathrm{~b}}=10.8 \mathrm{~Hz}, \mathrm{H}-6\right), 3.900\left(\mathrm{dd}, 1 \mathrm{H}, J_{5,6}=3.7, J_{6 \mathrm{a}, 6 \mathrm{~b}}=\right.$ $11.0 \mathrm{~Hz}, \mathrm{H}-6), 3.995$ (dd, $1 \mathrm{H}, J_{1,2}<1.0 \mathrm{~Hz}, J_{2,3}=3.2 \mathrm{~Hz}$, H-2 ${ }^{\mathrm{C}}$ ), 4.050-4.260 (m, 6H, H-2 $\left.{ }^{\mathrm{E}}, \mathrm{H}-5^{\mathrm{A}, \mathrm{C}, \mathrm{D}, \mathrm{E}}, 2 \mathrm{H}-6\right) 4.269$ $\left(\mathrm{m}, 1 \mathrm{H}, \mathrm{H}-5^{\mathrm{B}}\right), 4.350-4.437\left(\mathrm{~m}, 3 \mathrm{H}, \mathrm{H}-3^{\mathrm{A}}, \mathrm{CH}_{2}=\mathrm{CH}-\right.$ $\left.\mathrm{CH}_{2}-\right), 4.462\left(\mathrm{dd}, 1 \mathrm{H}, J_{1,2}<1.0 \mathrm{~Hz}, J_{2,3}=3.4 \mathrm{~Hz}, \mathrm{H}-2^{\mathrm{B}}\right)$, $4.627\left(\mathrm{~d}, 1 \mathrm{H}, J_{1,2}<1.0 \mathrm{~Hz}, \mathrm{H}-1^{\mathrm{B}}\right), 4.688\left(\mathrm{~d}, 1 \mathrm{H}, J_{1,2}\right.$ $\left.8.5 \mathrm{~Hz}, \mathrm{H}-1^{\mathrm{E}}\right), 4.795\left(\mathrm{t}, J_{1,2}=8.9 \mathrm{~Hz}, J_{2,3}=8.9 \mathrm{~Hz}, \mathrm{H}-2^{\mathrm{D}}\right)$, $4.914\left(\mathrm{~s}, \quad 1 \mathrm{H}, \quad \mathrm{H}-1^{\mathrm{C}}\right), \quad 5.011 \quad\left(\mathrm{t}, \quad 1 \mathrm{H}, \quad J_{3,4}=8.7 \mathrm{~Hz}\right.$, $\left.J_{4,5}=8.7 \mathrm{~Hz}, \mathrm{H}-4^{\mathrm{E}}\right), 5.070\left(\mathrm{~d}, 1 \mathrm{H}, J_{1,2}<1 \mathrm{~Hz}, \mathrm{H}-1^{\mathrm{A}}\right), 5.163$ $\left(\mathrm{dd}, 1 \mathrm{H}, J_{2,3}=3.2 \mathrm{~Hz}, J_{3,4}=10.1 \mathrm{~Hz}, \mathrm{H}-3^{\mathrm{C}}\right), 5.300-5.480(\mathrm{~m}$, $4 \mathrm{H}, J_{1,2}=8.8 \mathrm{~Hz}, J_{3,4}=9.8 \mathrm{~Hz}, \mathrm{H}-1^{\mathrm{D}}, \mathrm{H}-4^{\mathrm{D}}, \mathrm{CH}_{2}=\mathrm{CH}-$ $\left.\mathrm{CH}_{2}-\right), 5.493\left(\mathrm{t}, 1 \mathrm{H}, J_{3,4}=10.1 \mathrm{~Hz}, J_{4,5}=10.1 \mathrm{~Hz}, \mathrm{H}-4^{\mathrm{C}}\right)$, $5.520\left(\mathrm{dd}, 1 \mathrm{H}, J_{1,2}<1 \mathrm{~Hz}, J_{2,3}=3.2 \mathrm{~Hz}, \mathrm{H}-2^{\mathrm{A}}\right), 5.555(\mathrm{dd}$, $\left.1 \mathrm{H}, J_{2,3}=3.4 \mathrm{~Hz}, J_{3,4}=9.2 \mathrm{~Hz}, \mathrm{H}-3^{\mathrm{B}}\right), 5.677\left(\mathrm{t}, 1 \mathrm{H}, J_{3,4}=\right.$ $\left.10.4 \mathrm{~Hz}, J_{4,5}=10.4 \mathrm{~Hz}, \mathrm{H}-4^{\mathrm{A}}\right), 5.701\left(\mathrm{t}, 1 \mathrm{H}, J_{2,3}=9.3 \mathrm{~Hz}\right.$, $\left.J_{3,4}=9.3 \mathrm{~Hz}, \quad \mathrm{H}-3^{\mathrm{D}}\right), 5.783\left(\mathrm{t}, 1 \mathrm{H}, J_{2,3}=10.3 \mathrm{~Hz}, J_{3,4}=\right.$ $\left.10.3 \mathrm{~Hz}, \mathrm{H}-3^{\mathrm{E}}\right), 5.900-6.000\left(\mathrm{~m}, 1 \mathrm{H}, \mathrm{CH}_{2}=\mathrm{CH}_{-} \mathrm{CH}_{2}-\right)$, 7.250-8.307 (m, 48H, Ph). $\delta_{\mathrm{C}}\left(100 \mathrm{MHz}, \mathrm{CDCl}_{3}\right) 96.60$ $\left({ }^{1} J_{\mathrm{C}-1, \mathrm{H}-1}=170 \mathrm{~Hz}\right), \quad 98.20 \quad\left({ }^{1} J_{\mathrm{C}-1, \mathrm{H}-1}=173 \mathrm{~Hz}\right), \quad 98.50$ $\left({ }^{1} J_{\mathrm{C}-1, \mathrm{H}-1}=162 \mathrm{~Hz}\right), \quad 99.10 \quad\left({ }^{1} J_{\mathrm{C}-1, \mathrm{H}-1}=165 \mathrm{~Hz}\right), \quad 99.40$ $\left({ }^{1} J_{\mathrm{C}-1, \mathrm{H}-1}=174 \mathrm{~Hz}\right)$. MALDI-TOF MS: M+Na calcd: 2233, found: 2233.99; Anal. Calcd for $\mathrm{C}_{117} \mathrm{H}_{106} \mathrm{~N}_{2} \mathrm{O}_{42}$ : C, 63.53; H, 4.80. Found: C, 63.47; H, 4.76.

3.1.7. Allyl $2,3,4,6$-tetra- $O$-benzoyl- $\alpha$-D-mannopyranosyl-( $1 \rightarrow 3)$-[2,3,4,6-tetra- $O$-benzoyl- $\alpha$-D-mannopyranosyl- $(1 \rightarrow 6)]-2,4-d i-O$-benzoyl- $\alpha$-D-mannopyranoside (13). To a cooled solution $\left(0^{\circ} \mathrm{C}\right)$ of $3(1.5 \mathrm{~g}, 3.5 \mathrm{mmol})$ and 9 $(5.45 \mathrm{~g}, 7.36 \mathrm{mmol})$ in anhydrous $\mathrm{CH}_{2} \mathrm{Cl}_{2}(50 \mathrm{~mL})$ was added TMSOTf $(25 \mu \mathrm{L}, 0.14 \mathrm{mmol})$. The mixture was stirred at this temperature for $2 \mathrm{~h}$, and then quenched with $\mathrm{Et}_{3} \mathrm{~N}$ ( 2 drops). The solvents were evaporated in vacuo to give a residue, which was purified by silica gel column chromatography (petroleum ether-EtOAc, 1.5:1) to give trisaccharide 13 as a syrup $(4.72 \mathrm{~g}, 85 \%) ;[\alpha]_{\mathrm{D}}{ }^{25}=-271\left(c 1, \mathrm{CHCl}_{3}\right)$; $\nu_{\max }$ (liquid film): 3444, 2956, 1731, 1602, 1452, 1267, $1109,1070,710 \mathrm{~cm}^{-1} ; \delta_{\mathrm{H}}\left(400 \mathrm{MHz} \mathrm{CDCl}_{3}\right) 3.80(\mathrm{dd}$, $\left.1 \mathrm{H}, J_{5,6}<1 \mathrm{~Hz}, J_{6 \mathrm{a}, 6 \mathrm{~b}}=9.1 \mathrm{~Hz}, \mathrm{H}-6\right), 4.15-4.25(\mathrm{~m}, 2 \mathrm{H}$, 2H-6), 4.32-4.42 (m, 4H, H-5, 3H-6), 4.50-4.56 (m, 2H, 2H-5), 4.58-4.64 (m, 2H, $\left.\mathrm{CH}_{2}=\mathrm{CH}-\mathrm{CH}_{2}-\right), 4.70(\mathrm{dd}, 1 \mathrm{H}$, $\left.J_{2,3}=3.2 \mathrm{~Hz}, J_{3,4}=11.0 \mathrm{~Hz}, \mathrm{H}-3\right), 5.16\left(\mathrm{~d}, 1 \mathrm{H}, J_{1,2}=1.4 \mathrm{~Hz}\right.$, $\mathrm{H}-1), 5.20\left(\mathrm{~d}, 1 \mathrm{H}, J_{1,2}=1.2 \mathrm{~Hz}, \mathrm{H}-1\right), 5.30-5.35(\mathrm{~m}, 1 \mathrm{H}$, $\left.\mathrm{CH}_{2}=\mathrm{CH}-\mathrm{CH}_{2}-\right)$, 5.36-5.39 (m, 2H, H-1, H-2), 5.41$5.50\left(\mathrm{~m}, 1 \mathrm{H}, \mathrm{CH}_{2}=\mathrm{CH}-\mathrm{CH}_{2}-\right), 5.76\left(\mathrm{dd}, 1 \mathrm{H}, J_{2,3}=\right.$ $\left.3.1 \mathrm{~Hz}, J_{3,4}=12 \mathrm{~Hz}, \mathrm{H}-3\right), 5.77\left(\mathrm{dd}, 1 \mathrm{H}, J_{1,2}=1.6 \mathrm{~Hz}\right.$, $\left.J_{2,3}=3.3 \mathrm{~Hz}, \mathrm{H}-2\right), 5.79\left(\mathrm{dd}, 1 \mathrm{H}, J_{1,2}=1.8 \mathrm{~Hz}, J_{2,3}=3.1 \mathrm{~Hz}\right.$, $\mathrm{H}-2), 5.94\left(\mathrm{t}, 1 \mathrm{H}, J_{3,4}=10.4 \mathrm{~Hz}, J_{4,5}=10.4 \mathrm{~Hz}, \mathrm{H}-4\right), 6.00-$ $6.06\left(\mathrm{~m}, 3 \mathrm{H}, \mathrm{H}-3, \mathrm{H}-4, \mathrm{CH}_{2}=\mathrm{CH}-\mathrm{CH}_{2}-\right), 6.15(\mathrm{t}, 1 \mathrm{H}$, $\left.J_{3,4}=10.6 \mathrm{~Hz}, J_{4,5}=10.6 \mathrm{~Hz}, \mathrm{H}-4\right), 7.25-8.17(\mathrm{~m}, 50 \mathrm{H}$, $\mathrm{Ph}) . \quad \delta_{\mathrm{C}} \quad\left(100 \mathrm{MHz}, \mathrm{CDCl}_{3}\right) \quad 166.14, \quad 166.02, \quad 165.99$, $165.56,165.41,165.18,165.11$ (2C), 164.59, 164.57 (PhCO), $118.66\left(\mathrm{CH}_{2}=\mathrm{CH}-\mathrm{CH}_{2}-\right), 99.54,97.43,96.48$ $\left(3 \mathrm{C}-1,{ }^{1} J_{\mathrm{C}-1, \mathrm{H}-1}=170,170,173 \mathrm{~Hz}\right), 76.52,71.87,70.19$,

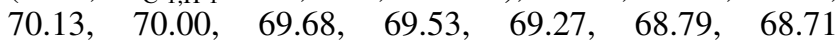
$\left(\mathrm{CH}_{2}=\mathrm{CH}-\mathrm{CH}_{2}-\right), 68.45,66.88,66.53,66.35,62.57$, 62.42. Anal. Calcd for $\mathrm{C}_{91} \mathrm{H}_{76} \mathrm{O}_{26}$ : C, 68.94; H, 4.80. Found: C, 68.97; H, 4.78. 
3.1.8. 2,3,4,6-Tetra-O-benzoyl- $\alpha$-D-mannopyranosyl$(1 \rightarrow 3)$-[2,3,4,6-tetra- $O$-benzoyl- $\alpha$-D-mannopyranosyl$(1 \rightarrow 6)$ ]-2,4-di- $O$-benzoyl- $\alpha$-D-mannopyranosyl trichloroacetimidate (14). A solution of $13(850 \mathrm{mg}, 0.54 \mathrm{mmol})$ in $90 \%$ aqueous acetic acid $(10 \mathrm{~mL})$ was added $\mathrm{NaOAc}$ (177 mg, $2.16 \mathrm{mmol}$ ) and $\mathrm{PdCl}_{2}$ (191 mg, $1.08 \mathrm{mmol}$ ). The mixture was stirred at rt overnight and then neutralized with saturated aqueous $\mathrm{NaHCO}_{3}$. The mixture was extracted with $\mathrm{CH}_{2} \mathrm{Cl}_{2}(3 \times 50 \mathrm{~mL})$ and the organic phase was concentrated. The residue was purified by a silica gel column chromatography to give hemiacetal trisaccharide intermediate as syrup. A solution of the above syrup, $\mathrm{CCl}_{3} \mathrm{CN}(0.22 \mathrm{~mL}$, 4 equiv.) and $\mathrm{DBU}(0.02 \mathrm{~mL})$ in dry $\mathrm{CH}_{2} \mathrm{Cl}_{2}(5 \mathrm{~mL})$ was stirred at $\mathrm{rt}$ for $2 \mathrm{~h}$. The solvents were removed in vacuo. The residue was purified by silica gel flash column chromatography to give trichloroacetimidate $14(752 \mathrm{mg}, 83 \%)$ as a white foam; $[\alpha]_{\mathrm{D}}{ }^{25}=-40\left(c 1, \mathrm{CHCl}_{3}\right) ; \delta_{\mathrm{H}}(400 \mathrm{MHz}$, $\left.\mathrm{CDCl}_{3}\right) 3.84\left(\mathrm{dd}, 1 \mathrm{H}, J_{5,6 \mathrm{a}}<1 \mathrm{~Hz}, J_{6 \mathrm{a}, 6 \mathrm{~b}}=9.2 \mathrm{~Hz}, \mathrm{H}-6 \mathrm{a}\right)$, $4.15\left(\mathrm{dd}, 1 \mathrm{H}, J_{5,6 \mathrm{~b}}=6.3 \mathrm{~Hz}, J_{6 \mathrm{a}, 6 \mathrm{~b}}=11.0 \mathrm{~Hz}, \mathrm{H}-6 \mathrm{~b}\right), 4.30-$ $4.37(\mathrm{~m}, 2 \mathrm{H}), 4.48-4.58(\mathrm{~m}, 5 \mathrm{H}), 4.73(\mathrm{dd}, 1 \mathrm{H}$, $\left.J_{2,3}=3.4 \mathrm{~Hz}, J_{3,4}=9.8 \mathrm{~Hz}, \mathrm{H}-3\right), 5.10\left(\mathrm{~d}, 1 \mathrm{H}, J_{1,2}=1.2 \mathrm{~Hz}\right.$, $\mathrm{H}-1), 5.38-5.41$ (m, 2H, H-1, H-2), 5.69-5.73 (m, 2H, H2, H-3), 5.90-5.95 (m, 2H), $6.01\left(\mathrm{t}, 1 \mathrm{H}, J_{3,4}=10.8 \mathrm{~Hz}\right.$, $\left.J_{4,5}=10.8 \mathrm{~Hz}, \mathrm{H}-4\right), 6.07 \quad\left(\mathrm{t}, 1 \mathrm{H}, \quad J_{3,4}=10.0 \mathrm{~Hz}, J_{4,5}=\right.$ $10.0 \mathrm{~Hz}, \mathrm{H}-4), 6.12\left(\mathrm{t}, 1 \mathrm{H}, J_{3,4}=10.0 \mathrm{~Hz}, J_{4,5}=10.0 \mathrm{~Hz}\right.$, $\mathrm{H}-4), 6.59$ (d, 1H, $\left.J_{1,2}=1.5 \mathrm{~Hz}, \mathrm{H}-1\right), 7.00-8.13(\mathrm{~m}, 50 \mathrm{H}$, $\mathrm{Ph}$ ), 9.00 (s, $1 \mathrm{H}, \mathrm{NH})$. Anal. Calcd for $\mathrm{C}_{90} \mathrm{H}_{72} \mathrm{Cl}_{3} \mathrm{NO}_{26}: \mathrm{C}$, 63.96; H, 4.26; found: C, 63.88; H, 4.24.

\subsubsection{Allyl 2,3,4,6-tetra- $O$-benzoyl- $\alpha$-D-mannopyrano-} syl-( $1 \rightarrow 3$ )-2,4-di- $O$-benzoyl- $\alpha$-D-mannopyranoside (15). To a stirred solution of $2(2 \mathrm{~g}, 2.55 \mathrm{mmol})$ in THF $(50 \mathrm{~mL})$ was added TBAF $(800 \mathrm{mg}, 2.55 \mathrm{mmol})$. The mixture was stirred at $\mathrm{rt}$ for $4 \mathrm{~h}$, then evaporated to dryness $\left(<40^{\circ} \mathrm{C}\right.$ water both) under reduced pressure. The residue was purified by silica gel column chromatography to give $\mathbf{4}$ as a white solid $(1.13 \mathrm{~g}, 66 \%)$. To a cooled solution $\left(-15^{\circ} \mathrm{C}\right)$ of 4 $(1.062 \mathrm{~g}, 1.58 \mathrm{mmol})$ and $9(1.23 \mathrm{~g}, 1.66 \mathrm{mmol})$ in dry $\mathrm{CH}_{2} \mathrm{Cl}_{2}(15 \mathrm{~mL})$ was added TMSOTf $(20 \mu \mathrm{L}, 0.11 \mathrm{mmol})$, and the mixture was stirred at this temperature for $40 \mathrm{~min}$, another portion of TMSOTf $(50 \mu \mathrm{L})$ was added. The reaction was warmed up to rt and stirred for $2 \mathrm{~h}$ further, then concentrated. The residue was purified by silica gel column chromatography to give $\mathbf{1 5}$ as a white solid (1.276 g, 80\%); $[\alpha]_{\mathrm{D}}{ }^{25}=-42\left(c 1.4, \mathrm{CHCl}_{3}\right) ; \nu_{\max }$ (liquid film): 3444, 2926, $1731,1602,1452,1317,1266,1110,1070,1028,711 \mathrm{~cm}^{-1}$; $\delta_{\mathrm{H}}\left(400 \mathrm{MHz}, \mathrm{CDCl}_{3}\right) 2.89(\mathrm{bs}, \mathrm{H}, \mathrm{OH}), 3.75-3.80(\mathrm{~m}, 2 \mathrm{H}$, 2H-6), 3.92-3.95 (m, 1H, H-5), 4.07 (ddd, 1H, $J_{4^{\prime}, 5^{\prime}}=$ $\left.10.0 \mathrm{~Hz}, J_{5^{\prime}, 6^{\prime} \mathrm{a}}=3.6 \mathrm{~Hz}, J_{5^{\prime}, 6^{\prime} \mathrm{b}}=2.4 \mathrm{~Hz}, \mathrm{H}-5^{\prime}\right), 4.18-4.25(\mathrm{~m}$, $\left.1 \mathrm{H}, \quad \mathrm{CH}_{2}=\mathrm{CH}-\mathrm{CH}_{2}-\right), 4.35 \quad\left(\mathrm{dd}, \quad 1 \mathrm{H}, \quad J_{5^{\prime}, 6^{\prime} \mathrm{a}}=3.6 \mathrm{~Hz}\right.$, $\left.J_{6^{\prime} \mathrm{a}, 6^{\prime} \mathrm{b}}=12.3 \mathrm{~Hz}, \mathrm{H}-6^{\prime} \mathrm{a}\right), 4.44-4.50\left(\mathrm{~m}, 1 \mathrm{H}, \mathrm{CH}_{2}=\mathrm{CH}-\right.$ $\left.\mathrm{CH}_{2}-\right), \quad 4.61\left(\mathrm{dd}, \quad 1 \mathrm{H}, \quad J_{5^{\prime}, 6^{\prime} \mathrm{b}}=2.4 \mathrm{~Hz}, \quad J_{6^{\prime} \mathrm{a}, 6^{\prime} \mathrm{b}}=12.3 \mathrm{~Hz}\right.$, H-6'b), 4.70 (dd, $\left.1 \mathrm{H}, J_{2,3}=3.5 \mathrm{~Hz}, J_{3,4}=9.8 \mathrm{~Hz}, \mathrm{H}-3\right), 5.15$ (d, $\left.1 \mathrm{H}, J_{1,2}=1.4 \mathrm{~Hz}, \mathrm{H}-1\right), 5.23-5.36(\mathrm{~m}, 3 \mathrm{H}, \mathrm{H}-2$ and $\left.\mathrm{CH}_{2}=\mathrm{CH}-\mathrm{CH}_{2}-\right), 5.41\left(\mathrm{~d}, 1 \mathrm{H}, J_{1^{\prime}, 2^{\prime}}=1.7 \mathrm{~Hz}, \mathrm{H}-1^{\prime}\right), 5.65-$ $5.69\left(\mathrm{~m}, 2 \mathrm{H}, \mathrm{H}-2^{\prime}, \mathrm{H}-3^{\prime}\right), 5.72\left(\mathrm{t}, 1 \mathrm{H}, J_{3,4}=10.0 \mathrm{~Hz}\right.$, $\left.J_{4,5}=10.0 \mathrm{~Hz}, \mathrm{H}-4\right), 5.89\left(\mathrm{~m}, 1 \mathrm{H}, \mathrm{CH}_{2}=\mathrm{CH}-\mathrm{CH}_{2}-\right), 6.02$ $\left(\mathrm{t}, 1 \mathrm{H}, J_{3^{\prime}, 4^{\prime}}=10.0 \mathrm{~Hz}, J_{4^{\prime}, 5^{\prime}}=10.0 \mathrm{~Hz}, \mathrm{H}-4^{\prime}\right), 7.19-8.25(\mathrm{~m}$, $30 \mathrm{H}, \mathrm{Ph}$ ). Anal. Calcd for $\mathrm{C}_{57} \mathrm{H}_{50} \mathrm{O}_{17}: \mathrm{C}, 67.99 ; \mathrm{H}, 4.97$. Found: C, 67.96; H, 4.99 .

3.1.10. Allyl 2,3,4,6-tetra- $O$-benzoyl- $\alpha$-D-mannopyranosyl- $(1 \rightarrow 6)$-[2,3,4,6-tetra- $O$-benzoyl- $\alpha$-D-mannopyrano- syl-( $1 \rightarrow 3)$ ]-2,4-di-O-benzoyl- $\alpha$-D-mannopyranosyl$(1 \rightarrow 6)$-[2,3,4,6-tetra- $O$-benzoyl- $\alpha$-D-mannopyranosyl$(1 \rightarrow 3)]-2,4-d i-O$-benzoyl- $\alpha$-D-mannopyranoside $\quad(16)$. To a cooled solution $\left(0^{\circ} \mathrm{C}\right)$ of $14(2 \mathrm{~g}, 1.18 \mathrm{mmol})$ and 15 $(1.13 \mathrm{~g}, 1.12 \mathrm{mmol})$ in dry $\mathrm{CH}_{2} \mathrm{Cl}_{2}(15 \mathrm{~mL})$ was added TMSOTf $(20 \mu \mathrm{L})$. The mixture was stirred at this temperature for $2 \mathrm{~h}$, then neutralized with $\mathrm{Et}_{3} \mathrm{~N}$ ( 2 drops), and concentrated. The residue was purified by silica gel column chromatography to give $\mathbf{1 6}$ as a syrup $(2.42 \mathrm{~g}, 85 \%)$; $[\alpha]_{\mathrm{D}}^{25}=-54\left(c 1.5, \mathrm{CHCl}_{3}\right) ; \nu_{\max }$ (liquid film): 3443 , $2957,1730,1603,1452,1266,1110,1070,711 ; \delta_{\mathrm{H}}$ $\left(400 \mathrm{MHz}, \mathrm{CDCl}_{3}\right) 3.44$ (br d, $\left.1 \mathrm{H}, J_{6 \mathrm{a}, 6 \mathrm{~b}}=9.2 \mathrm{~Hz}, \mathrm{H}-6\right)$, $3.82\left(\mathrm{dd}, 1 \mathrm{H}, J_{5,6 \mathrm{~b}}=1.7 \mathrm{~Hz}, J_{6 \mathrm{a}, 6 \mathrm{~b}}=9.2 \mathrm{~Hz}, \mathrm{H}-6\right), 4.01$ (dd, $\left.1 \mathrm{H}, J_{5,6 \mathrm{~b}}=6.8 \mathrm{~Hz}, J_{6 \mathrm{a}, 6 \mathrm{~b}}=12.0 \mathrm{~Hz}, \mathrm{H}-6\right), 4.10-4.15(\mathrm{~m}, 1 \mathrm{H}$, $\mathrm{H}-5), 4.20-4.53(\mathrm{~m}, 12 \mathrm{H}), 4.58\left(\mathrm{dd}, 1 \mathrm{H}, J_{5,6 \mathrm{~b}}=3.5 \mathrm{~Hz}\right.$, $\left.J_{6 \mathrm{a}, 6 \mathrm{~b}}=10.1 \mathrm{~Hz}, \mathrm{H}-6\right), 4.67\left(\mathrm{dd}, 1 \mathrm{H}, J_{2,3}=3.2 \mathrm{~Hz}, J_{3,4}=\right.$ $10.2 \mathrm{~Hz}, \mathrm{H}-3), 4.73\left(\mathrm{dd}, 1 \mathrm{H}, J_{2,3}=3.4 \mathrm{~Hz}, J_{3,4}=10.0 \mathrm{~Hz}\right.$, $\mathrm{H}-3), 4.78\left(\mathrm{~d}, 1 \mathrm{H}, J_{1,2}=1.5 \mathrm{~Hz}, \mathrm{H}-1\right), 5.17-5.19(\mathrm{~m}, 2 \mathrm{H}$, $\mathrm{H}-1$ and one proton of $\mathrm{CH}_{2}=\mathrm{CH}-\mathrm{CH}_{2}-$ ), 5.21 (d, $1 \mathrm{H}$, $\left.J_{1,2}=1.2 \mathrm{~Hz}, \mathrm{H}-1\right), 5.29-5.32(\mathrm{~m}, 3 \mathrm{H}, \mathrm{H}-1, \mathrm{H}-2$, and one proton of $\left.\mathrm{CH}_{2}=\mathrm{CH}-\mathrm{CH}_{2}-\right), 5.37\left(\mathrm{~d}, 1 \mathrm{H}, J_{1,2}=1.6 \mathrm{~Hz}\right.$, $\mathrm{H}-1), 5.44$ (dd, $\left.1 \mathrm{H}, J_{1,2}=2.0 \mathrm{~Hz}, J_{2,3}=3.0 \mathrm{~Hz}, \mathrm{H}-2\right), 5.57$ $\left(\mathrm{dd}, 1 \mathrm{H}, J_{1,2}=2.0 \mathrm{~Hz}, J_{2,3}=3.0 \mathrm{~Hz}, \mathrm{H}-2\right), 5.65(\mathrm{dd}, 1 \mathrm{H}$, $\left.J_{2,3}=2.8 \mathrm{~Hz}, J_{3,4}=10.0 \mathrm{~Hz}, \mathrm{H}-3\right), 5.69-5.74$ (m, 2H, H-2, $\mathrm{H}-3$ ), 5.82 (dd, $\left.1 \mathrm{H}, J_{1,2}=1.5 \mathrm{~Hz}, J_{2,3}=3.4 \mathrm{~Hz}, \mathrm{H}-2\right), 5.85-$ $6.01\left(\mathrm{~m}, 4 \mathrm{H}, \mathrm{H}-3,2 \mathrm{H}-4, \mathrm{CH}_{2}=\mathrm{CH}-\mathrm{CH}_{2}-\right), 6.04(\mathrm{t}, 1 \mathrm{H}$, $\left.J_{2,3}=10.1 \mathrm{~Hz}, \quad J_{3,4}=10.1 \mathrm{~Hz}, \mathrm{H}-4\right), 6.07$ (t, $1 \mathrm{H}, \quad J_{2,3}=$ $\left.10.8 \mathrm{~Hz}, J_{3,4}=10.8 \mathrm{~Hz}, \mathrm{H}-4\right), 6.08\left(\mathrm{t}, 1 \mathrm{H}, J_{2,3}=10.0 \mathrm{~Hz}\right.$, $\left.J_{3,4}=10.0 \mathrm{~Hz}, \quad \mathrm{H}-4\right), \quad 7.18-8.35 \quad(\mathrm{~m}, \quad 80 \mathrm{H}, \quad \mathrm{Ph}) ; \quad \delta_{\mathrm{C}}$ $\left(100 \mathrm{MHz}, \mathrm{CDCl}_{3}\right) \quad 166.13,166.10,166.08, \quad 166.05$, $165.92,165.75,165.57,165.47,165.17,165.14,165.06$, 165.02, 164.65 (2C), 164.62, 164.55 (16 PhCO), 118.41 $\left(\mathrm{CH}_{2}=\mathrm{CH}-\mathrm{CH}_{2}-\right), \quad 100.07,99.61,97.54,97.31,96.67$ $\left(5 \mathrm{C}-1,{ }^{1} J_{\mathrm{C}-1, \mathrm{H}-1}=171,172,172,173,174 \mathrm{~Hz}\right), 77.71$, $77.24,72.06,72.00,70.20,70.14,69.57,69.50,69.45$, $69.37,69.32,68.77,68.63,68.20,68.08,66.45,66.27$, 66.13, 62.50, 62.43. Anal. Calcd for $\mathrm{C}_{145} \mathrm{H}_{120} \mathrm{O}_{42}: \mathrm{C}$, 68.72; H, 4.74. Found: C, 68.79; H, 4.81.

3.1.11. Methyl 2,4-di-O-benzoyl-3-O-tert-butyldimethylsilyl- $\alpha$-D-mannopyranoside (17). 2.0 equiv. of $\mathrm{FeCl}_{3} \cdot 6 \mathrm{H}_{2} \mathrm{O}$ was added to a mixture of methyl 2,4-di-O-benzoyl-3- $O$ tert-butyldimethylsilyl-6- $O$-triphenylmethyl- $\alpha$-D-mannopyranoside $(3 \mathrm{~g}, 3.96 \mathrm{mmol})$ in $\mathrm{CH}_{2} \mathrm{Cl}_{2}(25 \mathrm{~mL})$. The mixture was stirred for $2 \mathrm{~h}$ at $\mathrm{rt}$, then diluted with more $\mathrm{CH}_{2} \mathrm{Cl}_{2}(50 \mathrm{~mL})$, washed with ice-cold water twice. The washings were re-extracted with $\mathrm{CH}_{2} \mathrm{Cl}_{2}$ $(20 \mathrm{~mL})$. The organic phase was combined, dried and concentrated, then subjected to column chromatography on silica gel with petroleum ether-EtOAc (4:1) as the eluent to give syrupy $17(1.78 \mathrm{~g}, 87 \%) ;[\alpha]_{\mathrm{D}}{ }^{25}=-255(c$ $0.8, \mathrm{CHCl}_{3}$ ); $\nu_{\max }$ (liquid film): 3443, 3066, 2927, 1319, $1452,1267,1110,1070,1030 ; \delta_{\mathrm{H}}\left(400 \mathrm{MHz}, \mathrm{CDCl}_{3}\right)$ $-0.08,0.06\left(2 \mathrm{~s}, 2 \times 3 \mathrm{H}, \mathrm{Si}\left(\mathrm{CH}_{3}\right)_{2}\right), 0.62(\mathrm{~s}, 9 \mathrm{H}, t-\mathrm{Bu})$, $2.50(\mathrm{bs}, 1 \mathrm{H}, \mathrm{OH}), 3.46\left(\mathrm{~s}, 3 \mathrm{H}, \mathrm{OCH}_{3}\right), 3.71(\mathrm{dd}, 1 \mathrm{H}$, $\left.J_{5,6 \mathrm{a}}=4.4 \mathrm{~Hz}, \quad J_{6 \mathrm{a}, 6 \mathrm{~b}}=12.6 \mathrm{~Hz}, \mathrm{H}-6 \mathrm{a}\right), \quad 3.75 \quad(\mathrm{dd}, 1 \mathrm{H}$, $\left.J_{5,6 \mathrm{~b}}=2.4 \mathrm{~Hz}, \quad J_{6 \mathrm{a}, 6 \mathrm{~b}}=12.6 \mathrm{~Hz}, \mathrm{H}-6 \mathrm{~b}\right), 3.88 \quad(\mathrm{ddd}, \quad 1 \mathrm{H}$, $\left.J_{4,5}=9.4 \mathrm{~Hz}, J_{5,6 \mathrm{a}}=4.4 \mathrm{~Hz}, J_{5,6 \mathrm{~b}}=2.4 \mathrm{~Hz}, \mathrm{H}-5\right), 4.44$ (dd, $\left.1 \mathrm{H}, \quad J_{2,3}=3.6 \mathrm{~Hz}, \quad J_{3,4}=9.4 \mathrm{~Hz}, \quad \mathrm{H}-3\right), 4.89 \quad(\mathrm{~d}, \quad 1 \mathrm{H}$, $\left.J_{1,2}=1.6 \mathrm{~Hz}, \mathrm{H}-1\right), 5.37\left(\mathrm{dd}, 1 \mathrm{H}, J_{1,2}=1.6 \mathrm{~Hz}, J_{2,3}=3.6 \mathrm{~Hz}\right.$, $\mathrm{H}-2), 5.55\left(\mathrm{t}, 1 \mathrm{H}, J_{3,4}=9.4 \mathrm{~Hz}, J_{4,5}=9.4 \mathrm{~Hz}, \mathrm{H}-4\right), 7.40-8.14$ (m, $10 \mathrm{H}, \mathrm{Ph})$. Anal. Calcd for $\mathrm{C}_{27} \mathrm{H}_{36} \mathrm{O}_{8} \mathrm{Si}: \mathrm{C}, 62.79 ; \mathrm{H}$, 6.98. Found: C, 62.82; H, 6.92. 
3.1.12. Methyl 2-O-acetyl-3,4,6-tri- $O$-benzoyl- $\alpha$-Dmannopyranosyl-( $1 \rightarrow$ 6)-2,4-di- $O$-benzoyl-3-O-tert-butyldimethylsilyl- $\alpha$-D-mannopyranoside (18). To a cooled solution $\left(0^{\circ} \mathrm{C}\right)$ of $17(850 \mathrm{mg}, 1.65 \mathrm{mmol})$ and $6(1.28 \mathrm{~g}$, $1.89 \mathrm{mmol})$ in anhydrous $\mathrm{CH}_{2} \mathrm{Cl}_{2}(15 \mathrm{~mL})$ was added TMSOTf $(25 \mu \mathrm{L}, 0.14 \mathrm{mmol})$. The mixture was stirred at this temperature for $1 \mathrm{~h}$, and then quenched with $\mathrm{Et}_{3} \mathrm{~N}$ (2 drops). The solvents were evaporated in vacuo to give a residue, which was purified by silica gel column chromatography (petroleum ether-EtOAc, 2:1) to give disaccharide $\mathbf{1 8}$ as a syrup $(1.43 \mathrm{~g}, 84 \%) ;[\alpha]_{\mathrm{D}}{ }^{25}=-103\left(c 1.1, \mathrm{CHCl}_{3}\right) ; \nu_{\max }$ (liquid film): $3440,2925,1730 ; \delta_{\mathrm{H}}\left(400 \mathrm{MHz}, \mathrm{CDCl}_{3}\right)$ $-0.11,0.06\left(2 \mathrm{~s}, 2 \times 3 \mathrm{H}, \mathrm{Si}\left(\mathrm{CH}_{3}\right)_{2}\right), 0.63(\mathrm{~s}, 9 \mathrm{H}, t-\mathrm{Bu}), 2.05$ $\left(\mathrm{s}, 3 \mathrm{H}, \mathrm{CH}_{3} \mathrm{CO}\right), 3.56\left(\mathrm{~s}, 3 \mathrm{H}, \mathrm{OCH}_{3}\right), 3.67(\mathrm{dd}, 1 \mathrm{H}$, $\left.J_{5,6 \mathrm{a}}=2.2 \mathrm{~Hz}, J_{6 \mathrm{a}, 6 \mathrm{~b}}=10.5 \mathrm{~Hz}, \mathrm{H}-6 \mathrm{a}\right), 4.04\left(\mathrm{dd}, 1 \mathrm{H}, J_{5,6 \mathrm{~b}}=\right.$ $\left.6.4 \mathrm{~Hz}, J_{6 \mathrm{a}, 6 \mathrm{~b}}=10.5 \mathrm{~Hz}, \mathrm{H}-6 \mathrm{~b}\right), 4.15\left(\mathrm{ddd}, 1 \mathrm{H}, J_{4,5}=9.6 \mathrm{~Hz}\right.$, $\left.J_{5,6 \mathrm{a}}=2.2 \mathrm{~Hz}, \quad J_{5,6 \mathrm{~b}}=6.4 \mathrm{~Hz}, \quad \mathrm{H}-5\right), \quad 4.32 \quad(\mathrm{ddd}, \quad 1 \mathrm{H}$, $\left.J_{4^{\prime}, 5^{\prime}}=9.6 \mathrm{~Hz}, \quad J_{5^{\prime}, 6 \mathrm{a}^{\prime}}<1.0 \mathrm{~Hz}, J_{5^{\prime}, 6 \mathrm{~b}^{\prime}}=5.2 \mathrm{~Hz}, \mathrm{H}-5^{\prime}\right), 4.38-$ 4.47 (m, 3H, H-3, 2H-6 $), 4.88\left(\mathrm{~d}, 1 \mathrm{H}, J_{1,2}=1.5 \mathrm{~Hz}, \mathrm{H}-1\right)$, $4.94\left(\mathrm{~d}, 1 \mathrm{H}, J_{1^{\prime}, 2^{\prime}}=1.7 \mathrm{~Hz}, \mathrm{H}-1^{\prime}\right), 5.40\left(\mathrm{dd}, 1 \mathrm{H}, J_{1,2}=1.5 \mathrm{~Hz}\right.$, $\left.J_{2,3}=3.6 \mathrm{~Hz}, \mathrm{H}-2\right), 5.44\left(\mathrm{dd}, 1 \mathrm{H}, J_{1^{\prime}, 2^{\prime}}=1.7 \mathrm{~Hz}, J_{2^{\prime}, 3^{\prime}}=3.1 \mathrm{~Hz}\right.$, $\left.\mathrm{H}-2^{\prime}\right), 5.67$ (dd, $\left.1 \mathrm{H}, J_{3,4}=10.0 \mathrm{~Hz}, J_{4,5}=9.6 \mathrm{~Hz}, \mathrm{H}-4\right), 5.82$ $\left(\mathrm{dd}, J_{2^{\prime}, 3^{\prime}}=3.1 \mathrm{~Hz}, J_{3^{\prime}, 4^{\prime}}=10.8 \mathrm{~Hz}, \mathrm{H}-3^{\prime}\right), 5.88(\mathrm{dd}, 1 \mathrm{H}$, $J_{3^{\prime}, 4^{\prime}}=10.8 \mathrm{~Hz}, J_{4^{\prime}, 5^{\prime}}=9.6 \mathrm{~Hz}, \mathrm{H}-4^{\prime}$ ), 7.36-8.11 (m, 25H, Ph). Anal. Calcd for $\mathrm{C}_{56} \mathrm{H}_{60} \mathrm{O}_{17} \mathrm{Si}: \mathrm{C}, 65.12 ; \mathrm{H}$, 5.81. Found: $\mathrm{C}$, 65.09; H, 5.88 .

\subsubsection{Methyl 3,4,6-tri- $O$-benzoyl- $\alpha$-D-mannopyranosyl-} $(1 \rightarrow$ 6)-2,4-di- $\boldsymbol{O}$-benzoyl- $\alpha$-D-mannopyranoside (19). To a cooled solution $\left(0^{\circ} \mathrm{C}\right)$ of $18(916 \mathrm{mg}, 0.89 \mathrm{mmol})$ in $\mathrm{CH}_{2} \mathrm{Cl}_{2}(7 \mathrm{~mL})$ and $\mathrm{MeOH}(7 \mathrm{~mL})$ was added acetyl chloride $(1 \mathrm{~mL})$. After $12 \mathrm{~h}$, another portion of $\mathrm{AcCl}(0.4 \mathrm{~mL})$ was added. The mixture was further stirred at $\mathrm{rt}$ for $24 \mathrm{~h}$, then diluted with $\mathrm{CH}_{2} \mathrm{Cl}_{2}(50 \mathrm{~mL})$, neutralized with $\mathrm{Et}_{3} \mathrm{~N}$ and washed with water. The organic phase was concentrated. The residue was purified on a silica gel column using EtOAc-petroleum ether (5:4) as the elutant gave 19 as a syrup $(544 \mathrm{mg}, 70 \%) ;[\alpha]_{\mathrm{D}}{ }^{25}=+25\left(c 0.5, \mathrm{CHCl}_{3}\right) ; \nu_{\max }$ (liquid film): 3444, 3067, 2926, 1731; $\delta_{\mathrm{H}}(400 \mathrm{MHz}$, $\left.\mathrm{CDCl}_{3}\right) 2.04$ (bs, 1H, OH), 2.31 (bs, $\left.1 \mathrm{H}, \mathrm{OH}\right), 3.52$ (s, 3H, $\left.\mathrm{OCH}_{3}\right), 3.75\left(\mathrm{dd}, 1 \mathrm{H}, J_{5,6 \mathrm{a}}=1.9 \mathrm{~Hz}, J_{6 \mathrm{a}, 6 \mathrm{~b}}=10.9 \mathrm{~Hz}, \mathrm{H}-6 \mathrm{a}\right)$, 4.08-4.28 (m, 5H, H-5, H-5', H-6b, H-6'a, H-6'b), 4.36 (bs, $\left.1 \mathrm{H}, \mathrm{H}-2^{\prime}\right), 4.40\left(\mathrm{dd}, 1 \mathrm{H}, J_{2,3}=3.5 \mathrm{~Hz}, J_{3,4}=10.1 \mathrm{~Hz}\right.$, $\mathrm{H}-3), 4.96$ (d, 1H, $\left.J_{1,2}=1.3 \mathrm{~Hz}, \mathrm{H}-1\right), 5.04$ (d, $1 \mathrm{H}$, $\left.J_{1^{\prime}, 2^{\prime}}=1.2 \mathrm{~Hz}, \mathrm{H}_{-1}^{\prime}\right), 5.44\left(\mathrm{dd}, 1 \mathrm{H}, J_{1,2}=1.3 \mathrm{~Hz}, J_{2,3}=3.5 \mathrm{~Hz}\right.$, $\mathrm{H}-2), 5.74\left(\mathrm{dd}, 1 \mathrm{H}, J_{2^{\prime}, 3^{\prime}}=3.2 \mathrm{~Hz}, J_{3^{\prime}, 4^{\prime}}=6.1 \mathrm{~Hz}, \mathrm{H}-3^{\prime}\right), 5.77(\mathrm{t}$, $\left.1 \mathrm{H}, J_{3,4}=10.1 \mathrm{~Hz}, J_{4,5}=10.1 \mathrm{~Hz}, \mathrm{H}-4\right), 7.25-8.17(\mathrm{~m}, 25 \mathrm{H}$, $\mathrm{Ph}$ ). Anal. Calcd for $\mathrm{C}_{48} \mathrm{H}_{44} \mathrm{O}_{16}: \mathrm{C}, 65.75 ; \mathrm{H}, 5.02$. Found: $\mathrm{C}$, 65.69; H, 5.10.

\subsubsection{Methyl 2-O-acetyl-3,4,6-tri- $O$-benzoyl- $\alpha$-D-manno-} pyranosyl- $(1 \rightarrow 2)-3,4,6$-tri- $O$-benzoyl- $\alpha$-D-mannopyranosyl- $(1 \rightarrow 6)$-[2- $O$-acetyl-3,4,6-tri- $O$-benzoyl- $\alpha$-D-mannopyranosyl-( $\rightarrow 3)$ ]-2,4-di- $O$-benzoyl- $\alpha$-D-mannopyranoside (20). To a cooled solution $\left(0^{\circ} \mathrm{C}\right)$ of $19(370 \mathrm{mg}$, $0.42 \mathrm{mmol})$ and $6(630 \mathrm{mg}, 0.93 \mathrm{mmol})$ in anhydrous $\mathrm{CH}_{2} \mathrm{Cl}_{2}(15 \mathrm{~mL})$ was added TMSOTf $(20 \mu \mathrm{L}, 0.11 \mathrm{mmol})$. The mixture was stirred at this temperature for $3 \mathrm{~h}$, and then quenched with $\mathrm{Et}_{3} \mathrm{~N}$ (2 drops). The solvents were evaporated in vacuo to give a residue, which was purified by silica gel column chromatography (petroleum ether-EtOAc, 2:3) to give tetrasaccharide $\mathbf{2 0}$ as a syrup (588 $\mathrm{mg}, 73 \%$ ); $[\alpha]_{\mathrm{D}}^{25}=-16\left(c 1.5, \mathrm{CHCl}_{3}\right) ; \nu_{\max }$ (liquid film): 3441 , $2928,1730,1452,1269,1108,1068,756,710 \mathrm{~cm}^{-1} ; \delta_{\mathrm{H}}$ $\left(400 \mathrm{MHz}, \mathrm{CDCl}_{3}\right)$ 1.87, $2.05\left(2 \mathrm{~s}, 6 \mathrm{H}, 2 \mathrm{CH}_{3} \mathrm{CO}\right), 3.48(\mathrm{~s}$,

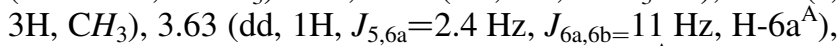
$3.98\left(\mathrm{dd}, 1 \mathrm{H}, J_{5,6 \mathrm{~b}}=5.8 \mathrm{~Hz}, J_{6 \mathrm{a}, 6 \mathrm{~b}}=11 \mathrm{~Hz}, \mathrm{H}-6 \mathrm{~b}^{\mathrm{A}}\right), 4.15(\mathrm{ddd}$, $\left.1 \mathrm{H}, J_{4,5}=9.5 \mathrm{~Hz}, J_{5,6 \mathrm{a}}=2.4 \mathrm{~Hz}, J_{5,6 \mathrm{~b}}=5.8 \mathrm{~Hz}, \mathrm{H}-5^{\mathrm{A}}\right), 4.26$ $\left(\mathrm{dd}, J_{1,2}=1.6 \mathrm{~Hz}, J_{2,3}=2.1 \mathrm{~Hz}, \mathrm{H}-2^{\mathrm{B}}\right), 4.35-4.56(\mathrm{~m}, 10 \mathrm{H}$, $\left.\mathrm{H}-3^{\mathrm{A}}, \mathrm{H}-5^{\mathrm{B}}, 5^{\mathrm{C}}, 5^{\mathrm{D}}, 2 \mathrm{H}-6^{\mathrm{B}}, 2 \mathrm{H}-6^{\mathrm{C}}, 2 \mathrm{H}-6^{\mathrm{D}}\right), 4.95(\mathrm{~d}, 1 \mathrm{H}$, $\left.J_{1,2}=1.5 \mathrm{~Hz}, \mathrm{H}-1^{\mathrm{A}}\right), 4.96\left(\mathrm{~d}, 1 \mathrm{H}, J_{1,2}=1.6 \mathrm{~Hz}, \mathrm{H}-1^{\mathrm{B}}\right), 5.12$ (dd, $1 \mathrm{H}, J_{1,2}=1.2 \mathrm{~Hz}, J_{2,3}=3.2 \mathrm{~Hz}, \mathrm{H}-2^{\mathrm{C}}$ ), 5.14 (d, $1 \mathrm{H}$, $\left.J_{1,2}=1.7 \mathrm{~Hz}, \mathrm{H}-1^{\mathrm{D}}\right), 5.16\left(\mathrm{~d}, 1 \mathrm{H}, J_{1,2}=1.2 \mathrm{~Hz}, \mathrm{H}-1^{\mathrm{C}}\right), 5.57$ $\left(\mathrm{dd}, 1 \mathrm{H}, J_{2,3}=3.2 \mathrm{~Hz}, J_{3,4}=9.9 \mathrm{~Hz}, \mathrm{H}-3^{\mathrm{C}}\right), 5.60(\mathrm{dd}, 1 \mathrm{H}$, $\left.J_{1,2}=1.7 \mathrm{~Hz}, \quad J_{2,3}=3.6 \mathrm{~Hz}, \mathrm{H}-2^{\mathrm{D}}\right), 5.66\left(\mathrm{dd}, 1 \mathrm{H}, J_{1,2}=\right.$ $\left.1.5 \mathrm{~Hz}, \quad J_{2,3}=3.8 \mathrm{~Hz}, \mathrm{H}-2^{\mathrm{A}}\right), 5.75\left(\mathrm{t}, 1 \mathrm{H}, J_{3,4}=9.9 \mathrm{~Hz}\right.$, $\left.J_{4,5}=9.9 \mathrm{~Hz}, \mathrm{H}-4^{\mathrm{C}}\right), 5.80\left(\mathrm{t}, 1 \mathrm{H}, J_{3,4}=9.5 \mathrm{~Hz}, J_{4,5}=9.5 \mathrm{~Hz}\right.$, $\left.\mathrm{H}-4^{\mathrm{A}}\right), 5.83-5.89\left(\mathrm{~m}, 3 \mathrm{H}, \mathrm{H}-3^{\mathrm{B}}, \mathrm{H}-3^{\mathrm{D}}, \mathrm{H}-4^{\mathrm{D}}\right), 5.96(\mathrm{t}, 1 \mathrm{H}$, $\left.J_{3,4}=9.8 \mathrm{~Hz}, J_{4,5}=9.8 \mathrm{~Hz}, \mathrm{H}-4^{\mathrm{B}}\right), 7.25-8.40(\mathrm{~m}, 55 \mathrm{H}, \mathrm{Ph})$. Anal. Calcd for $\mathrm{C}_{106} \mathrm{H}_{92} \mathrm{O}_{34}$ : C, 66.67; H, 4.82. Found: C, 66.71; H, 4.78.

\section{Acknowledgements}

This work was supported by CAS KIP-RCEES9904, $\mathrm{KJ} 952 \mathrm{~J}_{1} 510$, KJ952 $\mathrm{J}_{1} 561$, and NNSF of China (Projects 39970179 and 29972053).

\section{References}

1. Essentials of Glycobiology, Varki, A., Cummings, R., Esko, J., Freeze, H., Hart, G., Marth, J., Eds.; Cold Spring Harbor Laboratory: Cold Spring Harbor, New York, 1999.

2. Dwek, R. A. Chem. Rev. 1996, 96, 683-720.

3. (a) Rademann, J.; Geyer, A.; Schmidt, R. R. Angew. Chem. Int. Ed. 1998, 37, 1241. (b) Ogawa, T.; Katano, K.; Sasajima, K.; Matsui, M. Tetrahedron 1981, 37, 2779. (c) Ogawa, T.; Sasajima, K. Tetrahedron 1981, 37, 2787. (d) Guo, Z.; Nakahara, Y.; Ogawa, T. Tetrahedron Lett. 1997, 38, 4799. (e) Depre, D.; Duffels, A.; Green, L. G.; Lenz, R.; Ley, S. V.; Wong, C.-H. Chem. Eur. J. 1999, 5, 3326. (f) Dan, A.; Lergenmuller, M.; Amano, M.; Nakahara, Y.; Ogawa, T.; Ito, Y. Chem. Eur. J. 1998, 4, 2182. (g) Unverzagt, C.; Seifert, J. Tetrahedron Lett. 1997, 38, 7857. (h) Weiler, S.; Schmidt, R. R. Tetrahedron Lett. 1998, 39, 2292. (i) Garegg, P. J.; Oscarson, S.; Tiden, A. Carbohydr. Res. 1990, c3. (j) Wang, W.; Kong, F. Angew. Chem. Int. Ed. 1999, 38, 1247. (k) Becker, B.; Furneaux, R. H.; Reck, F.; Zubkov, O. A. Carbohydr. Res. 1999, 315, 148. (1) Ogawa, T.; Katano, K.; Matsui, M. Carbohydr. Res. 1978, 64, c3.

4. Du, Y.; Zhang, M.; Kong, F. Chinese Pat. Appl. 00107723.6.

5. Protection Groups in Organic Synthesis, Greene, T. W., Wuts, P. G. M., Eds.; Wiley: New York, 1991.

6. Du, Y.; Pan, Q.; Kong, F. Carbohydr. Res. 2000, 323, 28.

7. Ding, X.; Wang, W.; Kong, F. Carbohydr. Res. 1997, 303, 445.

8. Lowary, T. L.; Eichler, E.; Bundle, D. R. J. Org. Chem. 1995, 60, 7316.

9. EI-Sokkary, S. I.; Silwanis, B. A.; Nashed, M. A.; Paulsen, H. Carbohydr. Res. 1990, 203, 319.

10. Schmidt, R. R.; Michel, J. J. Carbohydr. Chem. 1985, 4, 141. 